\title{
A coupled volume-of-fluid/level-set method for simulation of two-phase flows on unstructured meshes
}

\author{
Néstor Balcázar ${ }^{a, *}$, Oriol Lehmkuhl ${ }^{\mathrm{a}, \mathrm{b}}$, Lluís Jofre ${ }^{\mathrm{a}}$, Joaquim Rigola ${ }^{\mathrm{a}}$, \\ Assensi Oliva ${ }^{\mathrm{a}, *}$ \\ ${ }^{a}$ Heat and Mass Transfer Technological Center (CTTC), Universitat Politècnica de \\ Catalunya - BarcelonaTech (UPC) ETSEIAT, Colom 11, 08222 Terrassa (Barcelona), \\ Spain.E-mail: cttc@upc.edu \\ ${ }^{b}$ Termo Fluids, S.L., Avda Jacquard 97 1-E, 08222 Terrassa (Barcelona), Spain. \\ E-mail: termofluids@termofluids.com
}

\begin{abstract}
This paper presents a methodology for simulation of two-phase flows with surface tension in the framework of unstructured meshes, which combines volume-of-fluid with level-set methods. While the volume-of-fluid transport relies on a robust and accurate polyhedral library for interface advection, surface tension force is calculated by using a level-set function reconstructed by means of a geometrical procedure. Moreover the solution of the fluid flow equations is performed through the fractional step method, using a finite-volume discretization on a collocated grid arrangement. The numerical method is validated against two- and three-dimensional test cases well established in the literature. Conservation properties of this method are shown to be excellent, while geometrical accuracy remains satisfactory even for the
\end{abstract}

\footnotetext{
*Corresponding author. Fax: +(34) 937398920

Email addresses: nestor@cttc.upc.edu (Néstor Balcázar), cttc@cttc.upc.edu (Assensi Oliva )
} 
most complex flows.

Keywords: unstructured meshes; level-set method; volume-of-fluid method; finite-volume method; two-phase flow

\section{Introduction}

Numerical simulation of two-phase flows is vital for many engineering and scientific applications, such as combustion, bubbly flow, boiling heat transfer, unit operations in chemical engineering, cooling of nuclear reactors among others. The accurate modeling of interfacial flows is challenging because of the discontinuity in material properties (e.g. density, viscosity), the necessity to account for surface tension force, and due to the fact that the geometry of the interface is not known a priory. For this kind of flows it is critical a precise computation of interfacial quantities such as curvature and normal, which are used to evaluate the surface tension. Errors in the calculated surface tension force will induce non-physical velocities, commonly known as spurious or parasitic currents [32], which can grow with time and so significantly degrade simulation results. Moreover, most of industrial applications are characterized by complex domains, therefore the use of unstructured meshes is advantageous.

In order to solve the aforementioned issues many numerical methods have been developed in the past decades. For instance: the front tracking (FT) method [47, 46], level set (LS) methods [30, 42, 29, 1], volume-of-fluid (VOF) methods [18, 48, 24], and hybrid VOF/LS methods [43, 49, 41, 28]. In these 
methods, two-phase flow is t reated a s a s ingle fl ow wi th th e de nsity and viscosity varying smoothly across the moving interface which is captured in an Eulerian framework (VOF, LS, CLSVOF, VOSET) or in a Lagrangian framework (FT). Although the idea behind these methods is similar, their numerical implementation may differ greatly. A review of advantages and disadvantages of these techniques in the context of simulation of multiphase flows w ith s harp i nterfaces is g iven in [ 48]. I n t he f ront-tracking method $[47,46]$, a stationary Eulerian grid is used for the fluid flow and the interface is tracked explicitly by a separate Lagrangian grid. This method is extremely accurate but also rather complex to implement due to the fact that dynamic re-meshing of the Lagrangian interface mesh is required [10]. Contrary to LS and VOF method, automatic merging of interfaces does not occur, and difficulties arise when multiple interfaces interact with each other as in coalescence and break-up. In the VOF method $[18,48,24]$, the interface is given implicitly by a color function, defined to be the fraction of volume within each cell of one of the fluids. In order to a dvect the VOF function, the interface needs to be reconstructed using a geometric technique [24]. An advantage of VOF method is the fact that accurate algorithms can be used to advect the interface (e.g. [24]), so that the mass is conserved, while still maintaining a sharp representation of the interfaces [43]. However a disadvantage of the VOF method is the fact that it is difficult to compute accurate curvatures from the volume fraction function used to represent the interface, because it presents a step discontinuity. In level-set (LS) methods $[30,42]$ the interface 
is represented by the zero-contour of a signed distance function. The evolution of this function in space and time is governed by an advection equation, combined with a special re-distancing algorithm. One of the advantage of the LS approach is the fact that the interface curvature can be accurately computed, while a disadvantage of this method is that the discrete solution of transport equations leads to numerical error in mass conservation of the fluid-phases. Recently, [29] has introduced a conservative level-set method (CLS) where mass conservation problem is greatly reduced, while an hyperbolic tangent function is employed as the level-set function. Moreover, this approach has been generalized to unstructured meshes by $[1,2,3]$, in the framework of finite-volume discretizations.

On the basis of the advantages and disadvantages of VOF and LS methods, it can be concluded that they are complementary, so it is an inevitable trend to develop new methods combining VOF and LS approaches. For instance, [43] presented a coupled level-set/volume-of-fluid (CLSVOF) method for computing 3D and axisymmetric incompressible two-phase flows. In the CLSVOF method the curvature was obtained via finite differences of the level set function which in turn is derived from the level set function and volumeof-fluid function. [50] present an adaptive coupled level-set/volume-of-fluid (ACLSVOF) method for interfacial flow simulations on two-dimensional unstructured triangular grids. Another CLSVOF method was implemented by [49] for the numerical simulations of interfacial flows in ship hydrodynamics, where the level set function is re-distanced based on the reconstructed inter- 
face with a geometric algorithm, whereas the interface jump conditions were handled by means of a ghost fluid methodology. This method was employed to simulate a gas bubble rising in a viscous liquid and a water drop impact onto a deep water pool. [41] have also presented a coupled volume-of-fluid and level set (VOSET) method, where a distance function is reconstructed from an iterative algorithm and interface is advected by the VOF method. The VOSET method was validated by performing two-dimensional simulations of rising bubbles and dam-break problems.

Despite those efforts, to the best of the author's knowledge most of the aforementioned coupled VOF/LS methods have been designed for regular cartesian meshes, so that their easiness of implementation, capability and accuracy on irregular unstructured meshes is still to be proven. Therefore, the present work is aimed at making progress in the direction of developing an accurate and robust coupled VOF/LS method for simulation of incompressible two-phase flows on two- and three dimensional unstructured meshes, including surface tension effects. Thus, unstructured meshes can be adapted to complex domains, enabling us an efficient mesh distribution in regions where interface resolution has to be maximized, which is in general hard to achieve on structured grids. In the present coupled VOF/LS method, an accurate VOF-PLIC method introduced by [24] is used to advect the interface, while the interface curvature and normals employed to evaluate the surface tension force are computed by using a level-set function. This LS function is reconstructed through a geometrical procedure, based on the computation 
of the minimum distances between cell centroids and the plane segments provided by the PLIC-VOF method introduced by [24], while the surface tension force is computed in the framework of the continuous surface force model introduced by [5]. Regarding the fluid flow, a classical fractional step method [7] is used to solve the incompressible Navier-Stokes equations, which are coupled with the VOF and LS functions. The Navier-Stokes equations have been discretized by means of the finite-volume method on a collocated unstructured grid arrangement, according to the work introduced in [1]. Numerical results are contrasted against numerical and experimental data from the literature.

The outline of this paper is as follows: A summary of the governing equations and numerical methods is given in Section 2. In Section 3 numerical experiments are presented in order to validate the coupled VOF/LS method implemented in this work. These numerical experiments include the simulation of the static droplet test case, two- and three-dimensional buoyant bubbles, co-axial coalescence of two bubbles, and deformation of a drop under shear flow. Finally, the conclusions are presented in Section 4.

\section{Governing equations and discretization}

\subsection{Incompressible two-phase flow}

The conservation of momentum and mass of two immiscible incompressible and Newtonian fluids is described by the Navier-Stokes equations defined 
on a spatial domain $\Omega$ with boundary $\partial \Omega$ :

$$
\frac{\partial}{\partial t}\left(\rho_{k} \mathbf{v}_{k}\right)+\nabla \cdot\left(\rho_{k} \mathbf{v}_{k} \mathbf{v}_{k}\right)=\nabla \cdot \mathbf{S}_{k}+\rho_{k} \mathbf{g} \quad \text { in } \Omega_{k}
$$

$$
\mathbf{S}_{k}=-p_{k} \mathbf{I}+\mu_{k}\left(\nabla \mathbf{v}_{k}+\left(\nabla \mathbf{v}_{k}\right)^{T}\right)
$$

$$
\nabla \cdot \mathbf{v}_{k}=0 \quad \text { in } \Omega_{k}
$$

Here, $\Omega=\Omega_{1} \cup \Omega_{2} \cup \Gamma, k=\{1,2\}$ denote the subdomains associated with the two different fluid phases, $\Gamma=\partial \Omega_{1} \cap \partial \Omega_{2}$ is the fluid interface, $\rho$ and $\mu$ denote the density and dynamic viscosity of the fluids, $\mathbf{v}$ is the velocity field, $\mathbf{g}$ is the gravity acceleration, $p$ is the pressure, $\mathbf{S}$ is the stress tensor and $\mathbf{I}$ is the identity tensor. Assuming no mass transfer between the fluids yields a continuous velocity condition at the interface:

$$
\mathbf{v}_{1}=\mathbf{v}_{2} \quad \text { in } \Gamma
$$

The jump in normal stresses along the fluid interface is balanced by the surface tension. Neglecting the variations of the surface tension coefficient $\sigma$ gives the following boundary condition for momentum conservation at the 
interface:

$$
\left(\mathbf{S}_{1}-\mathbf{S}_{2}\right) \cdot \mathbf{n}=\sigma \kappa \mathbf{n} \quad \text { in } \Gamma
$$

where $\mathbf{n}$ is the unit normal vector outward to $\partial \Omega_{1}$ and $\kappa$ is the interface curvature. Eqs. 1-3 and Eqs. 4-5 can be combined into a set of equations for a single fluid in $\Omega$, with a singular source term for the surface tension force at the interface $\Gamma[31,5,8]$ :

$$
\frac{\partial}{\partial t}(\rho \mathbf{v})+\nabla \cdot(\rho \mathbf{v} \mathbf{v})=-\nabla p+\nabla \cdot \mu\left(\nabla \mathbf{v}+(\nabla \mathbf{v})^{T}\right)+\rho \mathbf{g}+\sigma \kappa \mathbf{n} \delta_{\Gamma}
$$

$$
\nabla \cdot \mathbf{v}=0
$$

where $\mathbf{v}$ and $p$ denote the fluid velocity field and pressure, $\rho$ is the fluid density, $\mu$ is the dynamic viscosity, $\mathbf{g}$ is the gravitational acceleration the super-index $T$ represents the transpose operator, $\delta_{\Gamma}$ is a Dirac delta function concentrated at the interface $\Gamma, \sigma$ is the surface tension coefficient, $\kappa$ is the curvature of the interface and $\mathbf{n}$ denotes the unit normal vector on the interface. Physical properties change discontinuously across the interface:

$$
\begin{gathered}
\rho=\rho_{1} H_{1}+\rho_{2}\left(1-H_{1}\right) \\
\mu=\mu_{1} H_{1}+\mu_{2}\left(1-H_{1}\right)
\end{gathered}
$$


with $\rho_{1}, \rho_{2}$ and $\mu_{1}, \mu_{2}$ the densities and viscosities of the first and second fluids, respectively, whereas $H_{1}$ is the Heaviside step function that is one at fluid 1 and zero elsewhere. In the context of the present VOF/LS method, a volume averaged indicator function will be used in place of $H_{1}$, as is defined in Eq. 10 and Section 2.7.

\subsection{Volume-of-fluid method}

In the volume-of-fluid method an indicator function $f$ is used to track the interface,

$$
f(\mathbf{x}, t)= \begin{cases}1 & \text { if } \mathbf{x} \in \Omega_{1} \\ 0 & \text { if } \mathbf{x} \in \Omega_{2}\end{cases}
$$

with $\Omega_{1}$ and $\Omega_{2}$ the sub-domains occupied by the fluid 1 and 2 respectively. Discretely, the information effectively stored at the cell $\Omega_{P}$ is the volumeaveraged indicator function, namely the volume fraction:

$$
f_{P}=\frac{\int_{\Omega_{P}} f(\mathbf{x}, t) d V}{\int_{\Omega_{P}} d V}
$$

where $V$ is the volume of the cell $\Omega_{P}$. The advection equation for $f$ is given by:

$$
\frac{\partial f}{\partial t}+\mathbf{v} \cdot \nabla f=0
$$

where $\mathbf{v}$ is the fluid velocity. 
Following the work of [24], Eq. (11) is solved in two steps: first a geometric interface reconstruction is used to calculate the volumetric fluxes of one of the fluids across mesh cell faces, and second an advection step based on the reconstructed interface is performed by means of a first order Euler explicit scheme. The method here used reconstructs interfaces by means of piecewise planar approximations (PLIC). Therefore, a plane $\Pi$ (see Fig. 1a) is defined for each cell

$$
\mathbf{e}_{\Pi} \cdot \mathbf{x}_{\Pi}-d_{\Pi}=0
$$

Here $\mathbf{x}_{\Pi}$ is any position vector on the plane $\Pi, d_{\Pi}$ is determined such that the truncation between cell and plane satisfies a restriction for volume conservation [24], and $\mathbf{e}_{\Pi}$ is the plane normal computed according to the Youngs interface reconstruction method $[38,24]$, hence, $\mathbf{e}_{\Pi}=-\nabla f /\|\nabla f\|$ for each cell, with the gradient $\nabla f$ evaluated by means of the least-squares approach $[15,24,1]$. Volumes are advected in a single unsplit Lagrangian-Eulerian (LE) geometrical algorithm. The reader is referred to [24] for further details of the VOF-PLIC method used in the present work.

\subsection{Coupled volume-of-fluid/level-set method}

The main idea in the coupled VOF/LS methods $[43,41,49,28]$ is to take the advantages of both strategies, therefore, in the present formulation the mass losses are minimized through the use of a VOF-PLIC method introduced by [24], while a fine description of the interface curvature is kept by using the 
(a)

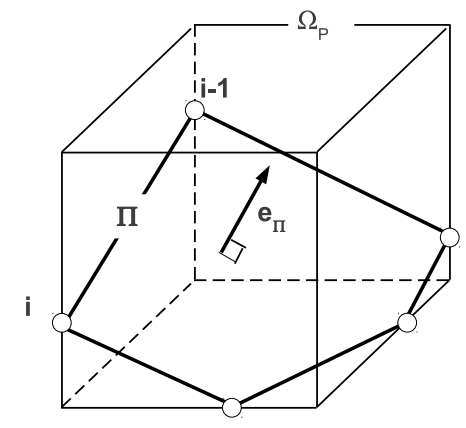

(b)

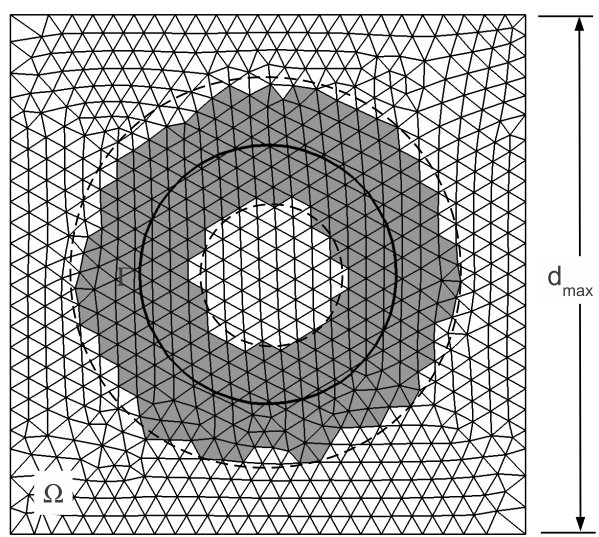

Figure 1: (a) Example of plane segment $\Pi$ at the hexahedral cell $\Omega_{P}$, obtained from the VOF-PLIC method [24]. (b) Example of two-dimensional flagged region around the interface.

level set method. From the geometrical information of the interface given by the VOF-PLIC method, a signed distance function is reconstructed following a geometric algorithm presented in Section 2.4. Then, the signed distance function is used to compute surface tension forces.

\subsection{Signed distance function construction, $d(\boldsymbol{x}, t)$}

The algorithm used to compute the signed distance function can be summarized as follows:

1. The signed distance function, $d\left(\mathbf{x}_{P}, t\right)$, is initialized at each mesh cell $P$ :

$$
d\left(\mathbf{x}_{P}, t\right)=\left\{\begin{array}{l}
d_{\max } \text { if } f\left(\mathbf{x}_{P}, t\right) \geq 0.5 \\
-d_{\max } \text { otherwise }
\end{array}\right.
$$


(a)

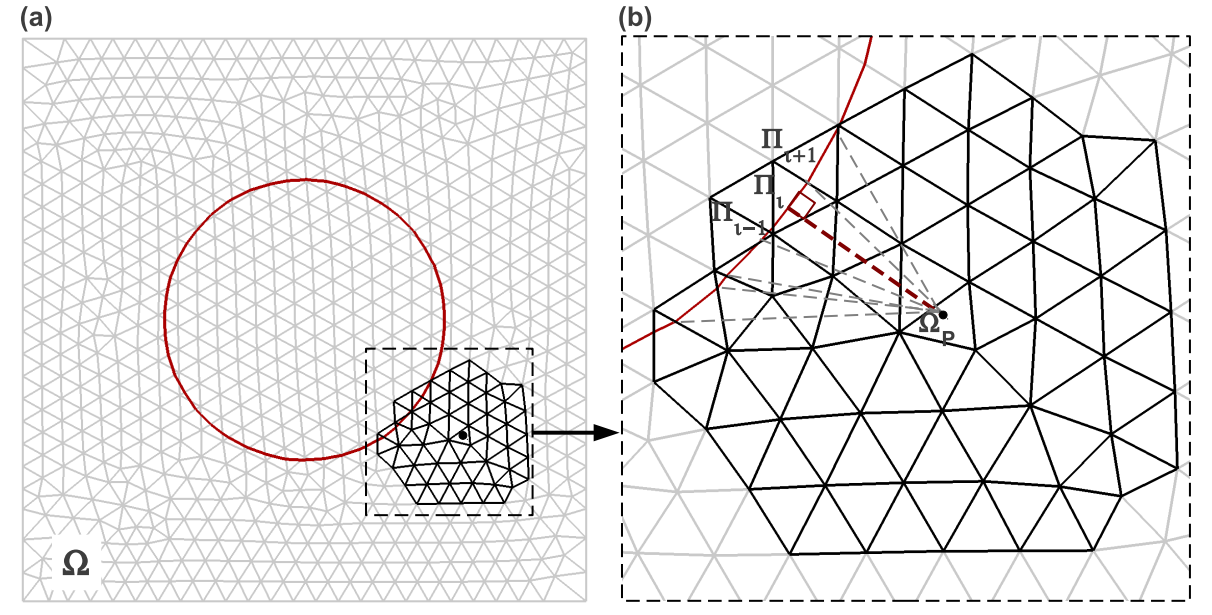

Figure 2: (a) Stencil of three cell layers around the current cell $\Omega_{P}$, with $\Omega_{P}$ included in the flagged region from Fig. 1b. (b) The dashed red line indicates the shortest line segment from $\mathbf{x}_{P}$ to $\left\{\Pi_{1}, \ldots, \Pi_{n}\right\}$ contained in the stencil of three cell layers around the cell $\Omega_{P}$. Here, $d\left(\mathbf{x}_{P}, t\right)=\min \left\{d_{P \rightarrow \Pi_{1}}^{\min }, \ldots, d_{P \rightarrow \Pi_{i}}^{\min }, \ldots, d_{P \rightarrow \Pi_{n}}^{\min }\right\}$

where $f\left(\mathbf{x}_{P}, t\right)$ is the VOF function defined in Eq. 10, and $d_{\max }$ is the maximum geometrical size of the space domain (see Fig. 1b).

2. In order to save computational time, only a set of cells near the interface are flagged to compute the distance function. This flagged zone is formed by the cells in a region of $\Delta_{\Gamma}=3 h$ width from the interface, as shown in Fig. 1b, where $h$ represents the characteristic size of the mesh cell.

3. The shortest distance $|d(\mathbf{x}, t)|$ is computed in the flagged region created in step 2, following the algorithm explained in section 2.5. For the sake of clarity, a two-dimensional example is illustrated in Fig. 2. 
4. The signed distance function is computed as follows:

$$
d\left(\mathbf{x}_{P}, 0\right)=\left\{\begin{array}{l}
-\left|d\left(\mathbf{x}_{P}, t\right)\right| \text { if } f\left(\mathbf{x}_{P}, t\right) \leqslant 0.5 \\
\left|d\left(\mathbf{x}_{P}, t\right)\right| \text { otherwise }
\end{array}\right.
$$

\subsection{Minimum distance from a cell-centroid to an interface-plane $\Pi$}

This procedure is applicable to compute the minimum distance from a cell-centroid $\mathbf{x}_{P}$ to an interface-plane $\Pi$ limited by a convex polygon which contains the coplanar points $\mathbf{x}_{1}, \ldots, \mathbf{x}_{i}, \mathbf{x}_{i+1}, \ldots, \mathbf{x}_{n}$, as shown in Fig. 3 where $n=5$. Following the example illustrated in Fig. 3, the algorithm is summarized as follows:

1. A set of planes $\Pi_{i-j}$ is defined, such that $\Pi_{i-j}$ is perpendicular to the interface-plane $\Pi$. In addition, $\Pi_{i, j}$ contains a pair of coplanar points $\left(\mathbf{x}_{i}, \mathbf{x}_{j}\right)$ for $(i, j)=(1,2), \ldots,(n-1, n),(n, 1)$, as shown in Fig. 3a, where $n=5$. If the next inequality is true for all the subindex $(i, j)$

$$
\left(\left(\mathbf{x}_{n}-\mathbf{x}_{P}\right) \times\left(\mathbf{x}_{1}-\mathbf{x}_{P}\right)\right) \cdot\left(\left(\mathbf{x}_{i}-\mathbf{x}_{P}\right) \times\left(\mathbf{x}_{j}-\mathbf{x}_{P}\right)\right) \geqslant 0
$$

then $\mathbf{x}_{P}$ is contained inside the region enclosed by the planes $\Pi_{1-2}, . ., \Pi_{n-1}$ (see Fig. 3a). Thus, the shortest distance from $\mathbf{x}_{P}$ to the interface-plane $\Pi$ is calculated as follows:

$$
\left|d\left(\mathbf{x}_{P}, t\right)\right|=\left|\left(\mathbf{x}_{P}-\mathbf{x}_{\Pi}\right) \cdot \mathbf{e}_{\Pi}\right|
$$


and the algorithm finished, otherwise it continues to the step 2. Here, $\mathbf{e}_{\Pi}$ is a unit vector perpendicular to the plane $\Pi$, while $\mathbf{x}_{\Pi}$ is a point contained in the plane $\Pi$.

2. A new set of planes $\Lambda_{i-j, k}$ is defined, such that $\Lambda_{i-j, k}$ is perpendicular to the interface-plane $\Pi, \Lambda_{i-j, k}$ is perpendicular to the vector $\Delta \mathbf{x}_{i, j}=$ $\mathbf{x}_{i}-\mathbf{x}_{j}$ with $(i, j)=\{(1,2),(2,3), \ldots,(n, 1)\}$, and $\Lambda_{i-j, k}$ contains a point $\mathbf{x}_{k}$ for $k=\{i, j\}$, as shown in Fig. 3b. If the next constraint is true

$$
0 \leq \frac{\left(\mathbf{x}_{P}-\mathbf{x}_{i}\right) \cdot\left(\mathbf{x}_{j}-\mathbf{x}_{i}\right)}{\left\|\mathbf{x}_{j}-\mathbf{x}_{i}\right\|} \leq 1
$$

then $\mathbf{x}_{P}$ is contained in the region enclosed by the planes $\Pi_{i, j}, \Lambda_{i-j, i}$ and $\Lambda_{i-j, j}$, as shown in Fig. 3b. In this case the minimum distance is given by

$$
\left|d\left(\mathbf{x}_{P}, t\right)\right|=\left\|\left(\mathbf{x}_{P}-\mathbf{x}_{i}\right)-\frac{\mathbf{x}_{j}-\mathbf{x}_{i}}{\left\|\mathbf{x}_{j}-\mathbf{x}_{i}\right\|}\left(\left(\mathbf{x}_{P}-\mathbf{x}_{i}\right) \cdot\left(\frac{\mathbf{x}_{j}-\mathbf{x}_{i}}{\left\|\mathbf{x}_{j}-\mathbf{x}_{i}\right\|}\right)\right)\right\|
$$

and the algorithm finished, otherwise it continues to the step 3.

3. In this case the minimum distance is given by

$$
\left|d\left(\mathbf{x}_{P}, t\right)\right|=\min \left\{|| \mathbf{x}_{P}-\mathbf{x}_{1}\left\|, \ldots,|| \mathbf{x}_{P}-\mathbf{x}_{n}\right\|\right\}
$$

as shown in Fig. 3c, where $n=5$. 


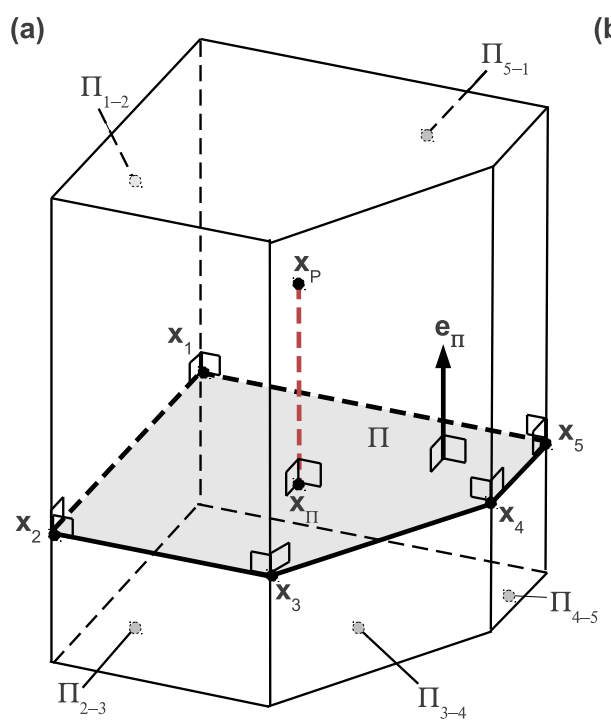

(b)

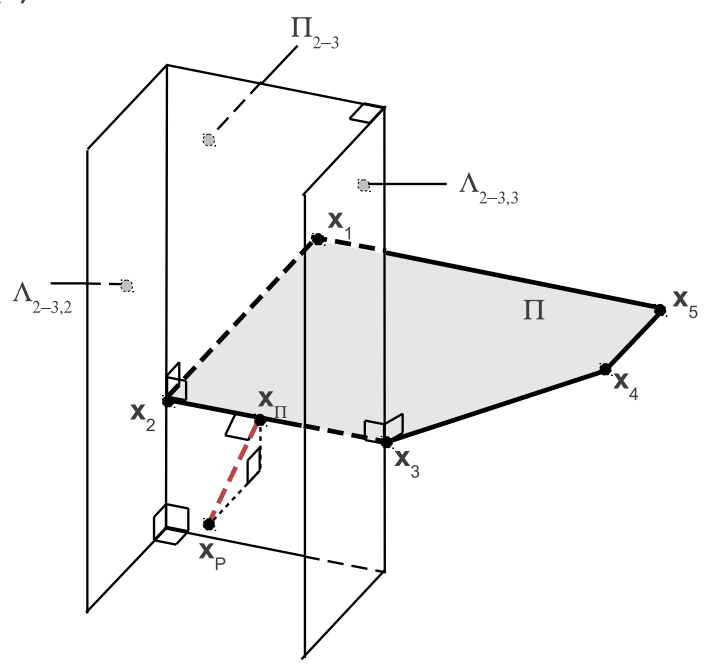

(c)

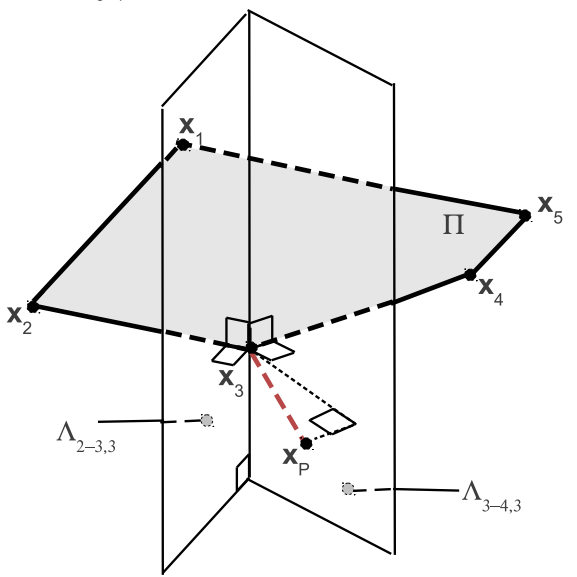

Figure 3: Minimum distance from $\mathbf{x}_{P}$ to the plane $\Pi_{i}$, in a three-dimensional framework. 


\subsection{Assessment of the distance function construction}

The accuracy of the distance function constructed by the algorithm introduced in Section 2.4 is measured. The distance function error $E_{2}$ is defined as

$$
E_{2}=\left(\frac{1}{n_{\text {cells }}} \sum_{P=1}^{n_{\text {cells }}}\left(d_{P}-d_{P}^{\text {exact }}\right)^{2}\right)^{1 / 2}
$$

where $n_{\text {cells }}$ is the number of cells contained in a flagged zone of $3 h$ width around the interface, and $P$ the index of the current cell $\Omega_{P}$. This test case has been carried out to perform the construction of the signed distance function around a cylindrical bubble of diameter $d_{b}=0.5$, centered in a unit square domain $2 d_{b} \times 2 d_{b}$ divided by triangular cells, as is illustrated in Fig. 4a. In addition, Fig. 4b shows $d(\mathbf{x}, t)$ around three bubbles, with $d_{b}=0.4$, centered in a unit square domain $2 d_{b} \times 2 d_{b}$ and triangular mesh with $h=d_{b} / 20$. Fig. 5 shows the error $E_{2}$ as function of the grid size $h$, with $p=1.22$ as the convergence order for the signed distance function, around the circle shown in Fig. 4a. Thus, from the present assessment it is demonstrated that the geometrical procedure introduced in Section 2.4 is robust enough to compute $d(\mathbf{x}, t)$ with high accuracy, even in presence of multiple interfaces.

\subsection{Surface tension force and regularization of physical properties}

Implementing surface tension in a numerical scheme involves two issues: the curvature $\kappa$ needs to be accurately calculated, and the resulting pres- 
(a)

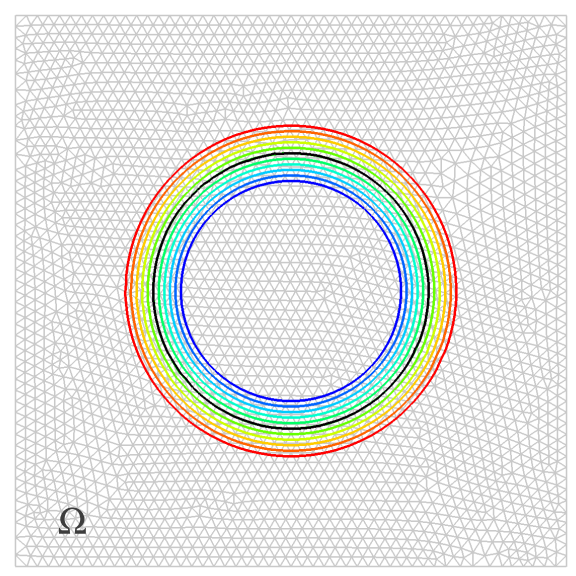

(b)

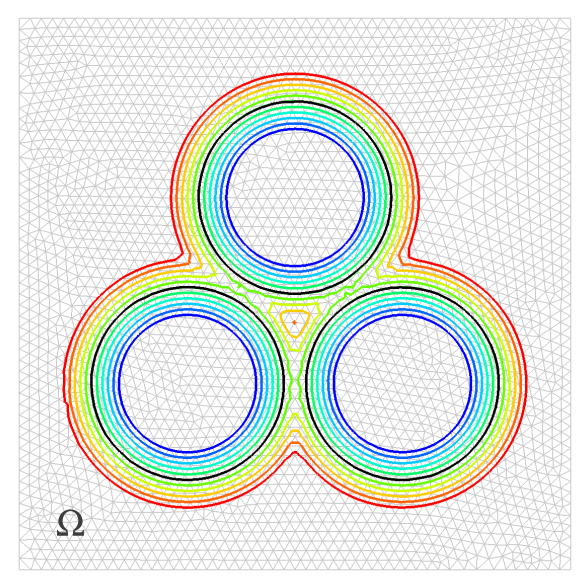

Figure 4: Interface position (black line) and constructed signed distance function (colour lines). (a) Cylindrical bubble of diameter $d_{b}$ on a triangular mesh with $h=d_{b} / 25$. (b) Three cylindrical bubbles of diameter $d_{b}$ on a triangular mesh with $h=d_{b} / 20$.

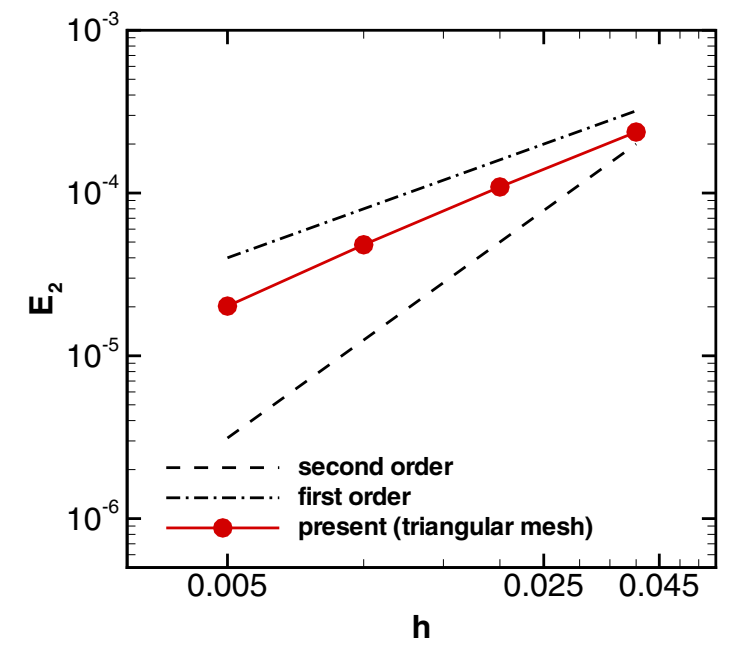

Figure 5: Error $\left(E_{2}\right)$ in distance function computation around a circle of diameter $d_{b}=0.5$ centered in a unit square domain (triangular mesh). For present results, $E_{2}=C h^{p}+$ $O\left(h^{p+1}\right)$, with $p=1.22$ the order of convergence of the algorithm illustrated in Section 2.4 . 
sure jump must be applied appropriately to the fluids. Since the present discretization will be based on the finite-volume integration of the NavierStokes Equations, Eq. (6), the aforementioned problems can be conveniently addressed through the continuous surface force model (CSF) introduced by [5]. Thus, the singular term, $\sigma \kappa \mathbf{n} \delta_{\Gamma}$, is converted to a volume force as follows

$$
\sigma \kappa \mathbf{n} \delta_{\Gamma}=\sigma \kappa(d) \nabla f
$$

where $\mathbf{n} \delta_{\Gamma}$ has been approximated by the gradient of the volume-averaged indicator function introduced in Eq. 10.

From the level-set function, $d(\mathbf{x}, t)$, the curvature $\kappa$ is obtained as the divergence of the interface unit normal $\mathbf{n}$ :

$$
\begin{aligned}
& \kappa=-\nabla \cdot \mathbf{n} \\
& \mathbf{n}=\left(\frac{\nabla d}{\|\nabla d\|}\right)
\end{aligned}
$$

To obtain a cell averaged value, the curvature is integrated over each finite volume $\Omega_{P}$ :

$$
\kappa_{P}=-\frac{1}{V_{P}} \int_{\Omega_{P}} \nabla \cdot \mathbf{n} d V
$$


Applying the Gauss theorem yields

$$
\kappa_{P}=-\frac{1}{V_{P}} \int_{S_{P}} \mathbf{n} \cdot d \mathbf{A}
$$

where $V_{P}$ and $S_{P}$ are the volume and surface of $\Omega_{P}$ respectively, while $\mathbf{A}$ is the area vector on $S_{P}$. Following the work of $[25,1]$, a wide and symmetric stencil is necessary for accurate evaluation of interface normals in the framework of unstructured meshes, therefore $\nabla d$ is computed using the least-squares method with vertex based stencil. The reader is referred to [1] for further details on the application of the least-squares method for gradient evaluation on unstructured meshes.

In the context of the present VOF/LS method, physical properties in Eq. 8 are regularized using a volume averaged indicator function, therefore density and viscosity fields are calculated as

$$
\begin{array}{r}
\rho=\rho_{1} f+\rho_{2}(1-f) \\
\mu=\mu_{1} f+\mu_{2}(1-f)
\end{array}
$$

with $f$ the VOF function introduced in Eq. 10.

\subsection{Solution procedure for the coupled VOF/LS method}

Following the work of [1], the Navier-Stokes equations, Eq. 6, have been discretized by means of the finite-volume method on a collocated unstructured grid. A central difference scheme is used to approximate the convective 
term of momentum equation, Eq. 6, unless otherwise stated; while diffusive terms are centrally differenced. A distance-weighted linear interpolation is used to find the cell face values of physical properties and interface normals, while gradients are computed at cell centroids by using the least-squares method [1]. The velocity-pressure coupling is solved by means of a classical fractional step projection method [7]. The solution procedure used in this work is summarized as follows:

1. Initialize $\mathbf{v}\left(\mathbf{x}_{P}, 0\right), f\left(\mathbf{x}_{P}, 0\right), d\left(\mathbf{x}_{P}, 0\right)$, physical properties and interface geometric properties.

2. The time increment $\Delta t$, which is limited by the CFL conditions and the stability condition for the capillary force [5], is calculated by

$$
\Delta t=C_{\Delta t} \min \left(\frac{h}{\|\mathbf{v}\|}, \frac{\rho h^{2}}{\mu},\left(\frac{h}{\|\mathbf{g}\|}\right)^{1 / 2}, h^{3 / 2}\left(\frac{\rho_{1}+\rho_{2}}{4 \pi \sigma}\right)^{1 / 2}\right)
$$

where $C_{\Delta t}=0.05$ for the current VOF/LS method.

3. The interface is advected by using the PLIC-VOF method introduced in [24] (see Section 2.2).

4. The signed distance function $d(\mathbf{x}, t)$ is calculated by the geometric algorithm given in Section 2.4.

5. The curvature is computed by Eq. 22 .

6. Physical properties $(\rho, \mu)$ are updated by Eq. 26 . 
7. An intermediate velocity $\mathbf{v}^{*}$ is evaluated by

$$
\frac{\rho \mathbf{v}^{*}-\rho \mathbf{v}^{n}}{\Delta t}=-\frac{3}{2} \mathbf{A}_{h}\left(\rho \mathbf{v}^{n}\right)+\frac{1}{2} \mathbf{A}_{h}\left(\rho \mathbf{v}^{n-1}\right)+\mathbf{D}_{h}\left(\mathbf{v}^{n}\right)+\rho \mathbf{g}+\sigma \kappa \nabla_{h}(\phi)
$$

where $\nabla_{h}$ represents the gradient operator, $D_{h}(\mathbf{v})=\nabla_{h} \cdot \mu\left(\nabla_{h} \mathbf{v}+\nabla_{h}^{T} \mathbf{v}\right)$ represents the diffusion operator, and $\mathbf{A}_{h}(\rho \mathbf{v})=\nabla_{h} \cdot(\rho \mathbf{v v})$ is the convective operator. For the temporal discretization, explicit AdamsBashforth scheme has been used.

8. The pressure field $p$ is computed by the Poisson equation

$$
\nabla_{h} \cdot\left(\frac{1}{\rho} \nabla_{h}\left(p^{n+1}\right)\right)=\frac{1}{\Delta t} \nabla_{h} \cdot\left(\mathbf{v}^{*}\right)
$$

Discretization of Eq. 29 leads to a linear system, which is solved by using a preconditioned conjugate gradient method.

9. The resulting velocity $\mathbf{v}^{*}$ from Eq. (28), does not satisfy the continuity Eq. (7). Therefore it is corrected by

$$
\mathbf{v}^{n+1}=\mathbf{v}^{*}-\frac{\Delta t}{\rho} \nabla_{h}\left(p^{n+1}\right)
$$

10. In order to avoid pressure-velocity decoupling when the pressure projection is made on collocated meshes $[40,12]$, a cell face velocity $\mathbf{v}_{f}$ is calculated so that $\nabla_{h} \cdot \mathbf{v}=0$ (see Eq. 7) at each control volume. 
Namely in discretized form:

$$
\mathbf{v}_{f}=\sum_{q \in\{P, F\}} \frac{1}{2}\left(\mathbf{v}_{q}^{n+1}+\frac{\Delta t}{\rho\left(\phi_{q}^{n}\right)}\left(\nabla_{h} p^{n+1}\right)_{q}\right)-\frac{\Delta t}{\rho_{f}}\left(\nabla_{h} p^{n+1}\right)_{f}
$$

where $P$ and $F$ are denoting the adjacent cell nodes to the face $f$.

11. Repeat steps 2-10 until time step required.

The numerical algorithms explained in this work have been implemented in the framework of a parallel $\mathrm{C}++$ code called TermoFluids [26]. The reader is referred to [1] for technical details of the spatial and temporal discretizations of the Navier-Stokes equations on collocated unstructured grids.

\section{Numerical experiments}

\subsection{Static drop}

The first test case is the verification of the stationary Laplace solution for a circular drop with diameter $d_{d}$. In the absence of viscous, gravitational or external forces, the circular interface with surface tension should remain at rest with the pressure jump at the interface exactly balancing the surface tension force (Laplace's law):

$$
\Delta P_{\text {exact }}=\sigma \kappa_{\text {exact }}
$$

where, the exact curvature is given by $\kappa_{\text {exact }}=2 / d_{d}$ for a circular drop. The correct solution is a zero velocity field and a pressure field that rises from 
(a)

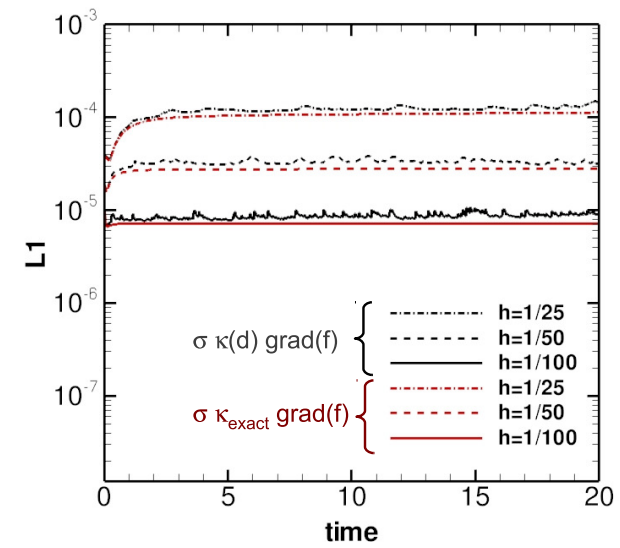

(b)

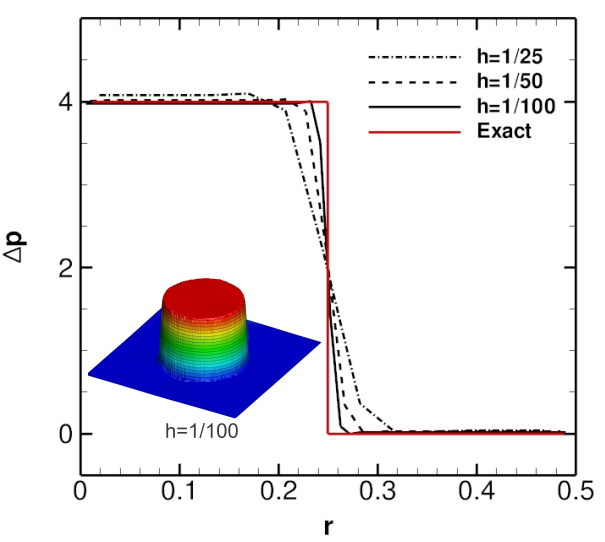

Figure 6: (a) Time evolution of spurious velocities. (b) Pressure profile for different grids, and pressure distribution on $\Omega$ for $h=1 / 100$.

a constant value of $p_{\text {out }}=p_{0}$ outside the drop to a value $p_{\text {in }}=p_{0}+2 \sigma / d_{d}$ inside the drop. However, at a discretized level, the accurate calculation of the curvature and the balance between the surface tension and pressure jump are not trivial problems, and, as a result spurious currents arise.

The computational domain is a square having side lengths of $2 d_{d}$ units, where $d_{d}=0.5$ is the diameter of a bubble positioned at the center of the domain. The coefficient of surface tension, and the viscosity inside and also outside the bubble were all set unity while the densities were given a magnitude of $10^{4}$. This corresponds to a Laplace number $L a=d_{d} \sigma \rho \mu^{-2}=5000$, which has been also used by [20]. Present test cases are solved on unstructured meshes of triangular element type, as is summarized in Table 1.

For the sake of comparison, the numerical jump in pressure is evaluated 


\begin{tabular}{cccc}
\hline Mesh name & Number of cells & Grid size $(h)$ & Cell geometry \\
\hline$M_{1}$ & $1.44 \times 10^{3}$ & $1 / 25$ & triangular \\
$M_{2}$ & $5.81 \times 10^{3}$ & $1 / 50$ & triangular \\
$M_{3}$ & $2.28 \times 10^{4}$ & $1 / 100$ & triangular \\
\hline
\end{tabular}

Table 1: Mesh parameters used in two-dimensional static droplet.

\begin{tabular}{ccccccc}
\hline & & $\sigma \kappa(d) \nabla f$ & & & $\sigma \kappa_{\text {exact }} \nabla f$ & \\
\cline { 7 - 7 } \cline { 6 - 7 } Mesh & $h$ & $L_{1}(\mathbf{v})$ & $E(\Delta p)$ & & $L_{1}(\mathbf{v})$ & $E(\Delta p)$ \\
\hline$M_{1}$ & $1 / 25$ & $1.30 \times 10^{-4}$ & 0.02433 & & $1.12 \times 10^{-4}$ & 0.02508 \\
$M_{2}$ & $1 / 50$ & $3.19 \times 10^{-5}$ & 0.00651 & & $2.79 \times 10^{-5}$ & 0.00804 \\
$M_{3}$ & $1 / 100$ & $8.82 \times 10^{-6}$ & 0.00215 & & $7.14 \times 10^{-6}$ & 0.00176 \\
$\mathrm{p} \approx$ & & 1.94 & 1.75 & & 1.99 & 1.92 \\
\hline
\end{tabular}

Table 2: Errors and convergence order $(\mathrm{p})$ for the dimensionless velocity and pressure using the VOF/LS method. Here, $\left\{L_{1}(\mathbf{v}), E(\Delta p)\right\}=C h^{p}+O\left(h^{p+1}\right)$ with $p$ the order of convergence.

as follows:

$$
E(\Delta p)=\frac{\left|p_{\text {in }}-p_{\text {out }}-2 \sigma / d_{d}\right|}{2 \sigma / d_{d}}
$$

where $p_{i n}$ is the pressure inside the drop which corresponds to the maximun pressure on $\Omega$, and $p_{\text {out }}$ is the outside pressure which corresponds to the minimum pressure on $\Omega$. Moreover, in order to measure the error in velocity, the following $L_{1}$ error norm is used:

$$
L_{1}(\mathbf{v})=\frac{1}{N_{\text {cells }}} \sum_{k}^{N_{\text {cells }}}\left(\mathbf{v}_{k} \cdot \mathbf{v}_{k}\right)^{1 / 2} \frac{\mu}{\sigma}
$$

which is computed on the whole of the spatial domain $\Omega$.

Table 2 shows the errors $E(\Delta p)$ and $L_{1}(\mathbf{v})$ for different grid sizes $(h)$. As with all Eulerian interface tracking methods there are spurious currents 
present, however, it can be observed from Fig. 6a that the parasitic currents measured by $L_{1}(\mathbf{v})$ are quite small in magnitude compared with other methods reported in the literature [51, 20], moreover its magnitude tends to a steady state as the time advances. In addition, Fig. 6a and Table 2 show a comparison of $L_{1}(\mathbf{v})$ calculated by the present surface tension model, $\sigma \kappa(d) \nabla f$, against the same model with an exact curvature. It is observed a very slight difference between these results, which confirms the accuracy of the method used to calculate the curvature. Regarding the pressure jump, Fig. 6b and Table 2 illustrate how well the computed pressure fulfilled the Young-Laplace law, Eq. 32, furthermore $E(\Delta p)$ decreased very rapidly with mesh refinement as is illustrated in Fig. 6b. Finally, data reported in Table 2 have been adjusted by the least-squares method to the function $\left\{L_{1}(\mathbf{v}), E(\Delta p)\right\}=C h^{p}$ to estimate the order of convergence $p$. The aforementioned results confirm that surface tension model was implemented correctly and it produces accurate results.

\subsection{Two-dimensional rising bubble}

This test case has been solved by $[19,20]$ in order to determine quantitative reference solutions for the buoyancy-driven motion of a two-dimensional bubble rising in an initially quiescent liquid. The computational setup is illustrated in Fig. 7, where a cylindrical bubble of diameter $d_{b}=0.5$ is centered in the lower half of a rectangular domain $\Omega=\left[0,2 d_{b}\right] \times\left[0,4 d_{b}\right]$. Non-slip boundary condition is applied at the top and bottom boundaries, and free 


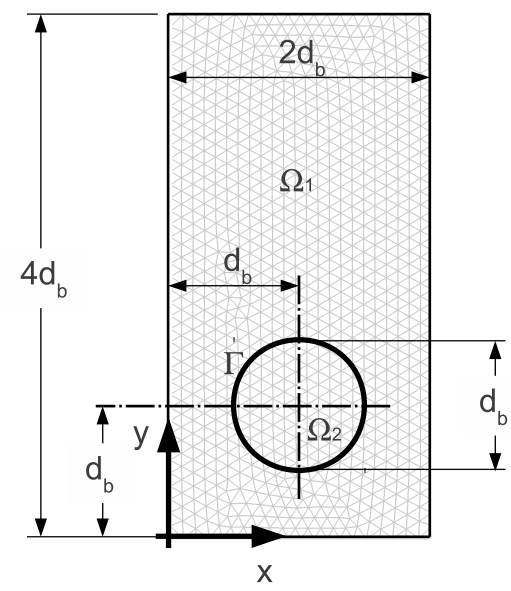

Figure 7: Computational setup for simulation of two-dimensional rising bubble.

slip boundary condition on the vertical walls. The physical properties of the fluid phases are summarized in Table 3 , where the subscript 1 is used for the continuous fluid phase, $\Omega_{1}$, while the subscript 2 is assigned to the lighter fluid in the bubble, $\Omega_{2}$.

\begin{tabular}{cccccccc}
\hline$\rho_{1}$ & $\rho_{2}$ & $\mu_{1}$ & $\mu_{2}$ & $g$ & $\sigma$ & $\rho_{1} / \rho_{2}$ & $\mu_{1} / \mu_{2}$ \\
\hline 1000 & 100 & 10 & 1 & 0.98 & 24.5 & 10 & 10 \\
\hline
\end{tabular}

Table 3: Physical parameters defining the two dimensional rising bubble test case.

For the sake of comparison, the benchmark quantities are defined as follows

$$
\left\{\begin{array}{l}
v_{c}=\frac{\int_{\Omega_{2}} \mathbf{v} \cdot \mathbf{e}_{y} d V}{\int_{\Omega_{2}} d V}, \\
y_{c}=\frac{\int_{\Omega_{2}} \mathbf{x} \cdot \mathbf{e}_{y} d V}{\int_{\Omega_{2}} d V}, \\
\zeta=\frac{\text { perimeter of area-equivalent circle }}{\text { perimeter of bubble }}=\frac{\pi d_{b}^{2}}{\int_{\Omega}\|\nabla f(\mathbf{x}, t)\| d V} .
\end{array}\right.
$$




\begin{tabular}{cccc}
\hline Mesh name & Number of cells & Grid size $(h)$ & Cell geometry \\
\hline$M_{1}$ & $2.91 \times 10^{4}$ & $d_{b} / 40$ & triangular \\
$M_{2}$ & $6.59 \times 10^{4}$ & $d_{b} / 60$ & triangular \\
$M_{3}$ & $1.17 \times 10^{5}$ & $d_{b} / 80$ & triangular \\
$M_{4}$ & $1.83 \times 10^{5}$ & $d_{b} / 100$ & triangular \\
\hline
\end{tabular}

Table 4: Mesh parameters used in two-dimensional rising bubble.

where $v_{c}$ is the rise velocity, $\mathbf{e}_{y}$ is a unit vector parallel to the $y-a x i s, y_{c}$ is the bubble centroid, $\zeta$ is the bubble circularity which takes the value 1 for a perfect circular bubble and values less than unity as the bubble is deformed.

Numerical simulations were carried out on unstructured meshes of triangular element type, with grid size $h=\{d / 40, d / 60, d / 80, d / 100\}$, as is illustrated in Fig. 7 and summarized in Table 4.

Fig. 8 shows the time evolution of the benchmark quantities defined in Eq. 35 for different grid resolutions. Moreover, it can be observed that computations performed by means of the VOF/LS method are in close agreement with the data reported by $[19,20]$ using a finite element level-set method. Furthermore, Fig. 8d shows the predicted bubble shapes for different times, and the streamlines calculated at steady state on the mesh $M_{4}$. The bubble, being initially circular, is horizontally stretched while it develops a slight dimple in the base which leads to a change in the interface curvature, to finally get an stable ellipsoidal shape because the action of surface tension force. Table 5 shows the inflection point of minimum circularity, center of mass $y_{c}(t=3)$ and maximum rise velocity $\left(v_{c}^{\max }\right)$, computed on the meshes $M_{2}$ and $M_{4}$, which illustrates that present results are in close agreement with benchmark data reported by [20]. In addition, present computations are also 
(a)

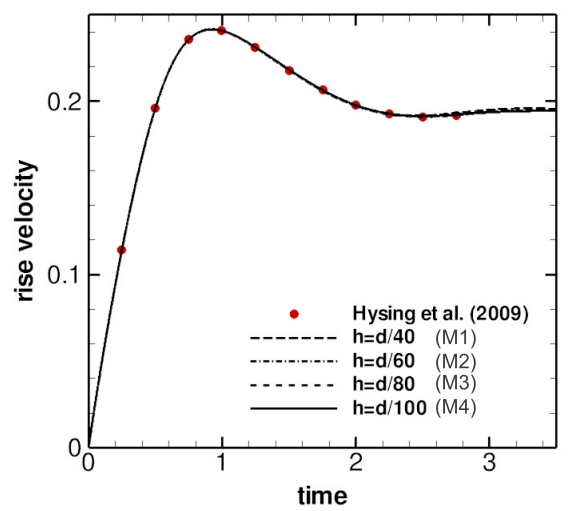

(c)

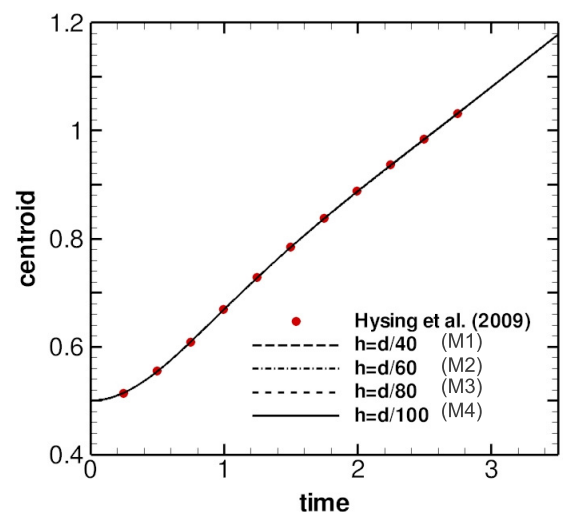

(b)

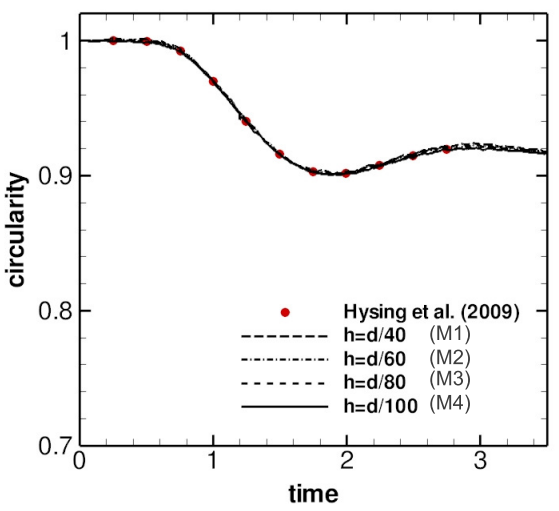

(d)

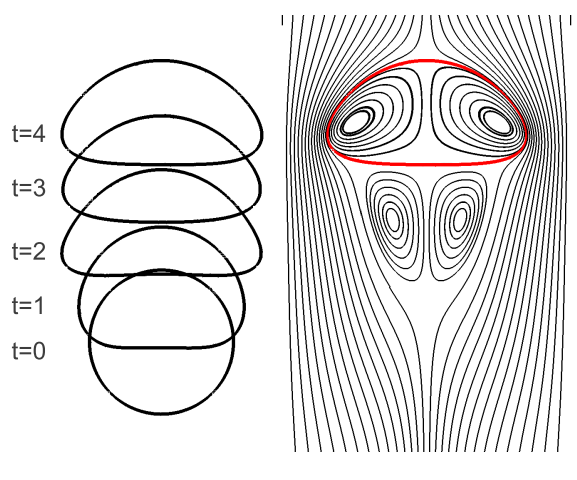

Figure 8: Two-dimensional rising bubble. (a) Rise velocity $\left(v_{c}\right)$. (b) Circularity $(\zeta)$. (c) Bubble centroid $\left(y_{c}\right)$. (d) Bubble shapes and streamlines calculated on the mesh $M_{4}$. 


\begin{tabular}{llllll}
\hline & \multicolumn{2}{l}{$h=d_{b} / 40$} & & \multicolumn{2}{l}{$h=d_{b} / 80$} \\
\cline { 2 - 3 } \cline { 5 - 6 } & {$[20]$} & VOF $/$ LS & & {$[20]$} & VOF $/$ LS \\
\hline$\zeta_{\text {min }}$ & 0.9014 & 0.9017 & & 0.9014 & 0.9005 \\
$\left.t\right|_{\zeta=\zeta_{\text {min }}}$ & 1.8734 & 1.8881 & & 1.9070 & 1.8934 \\
$v_{c}^{\text {max }}$ & 0.2418 & 0.2414 & & 0.2419 & 0.2414 \\
$\left.t\right|_{v_{c}=v_{c}^{\max }}$ & 0.9375 & 0.9190 & & 0.9281 & 0.9260 \\
$y_{c}(t=3)$ & 1.0810 & 1.0813 & & 1.0812 & 1.0809 \\
\hline
\end{tabular}

Table 5: Minimum circularity $\left(\zeta_{\min }\right)$ and maximum rise velocity $\left(v_{c}^{\max }\right)$, with corresponding incident times, and the final position of the center of mass. Present computations compared against Hysing et al. [20].

consistent with numerical simulation reported by [1] using the conservative level-set method.

\subsection{Three-dimensional buoyant bubbles}

This section is devoted to prove the stability and accuracy of the present VOF/LS method by performing simulations of three-dimensional rising bubbles for a wide range of flow conditions. The spatial domain is a cylinder with dimensions $\left(D_{\Omega}, H_{\Omega}\right)=(8 d, 8 d)$, where $d$ is the initial bubble diameter, $H_{\Omega}$ is the cylinder height and $D_{\Omega}$ is the cylinder diameter, as is illustrated in Fig. 9a. According to empirical studies of [17] the terminal rise velocity can be estimated through a semi-empirical relation:

$$
\frac{U_{T}}{U_{T}^{\infty}} \approx 1-\left(\frac{d}{D_{\Omega}}\right)^{2}
$$

where $U_{T}^{\infty}$ is defined as the terminal rise velocity in a domain of infinite extension. Moreover, numerical studies of [22] confirm the empirical findings of [17]. Indeed, given the selected domain, it is expected that the effect of 
(a)

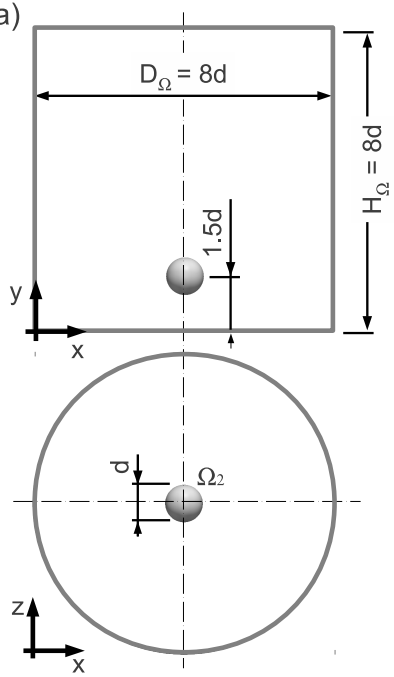

(b)

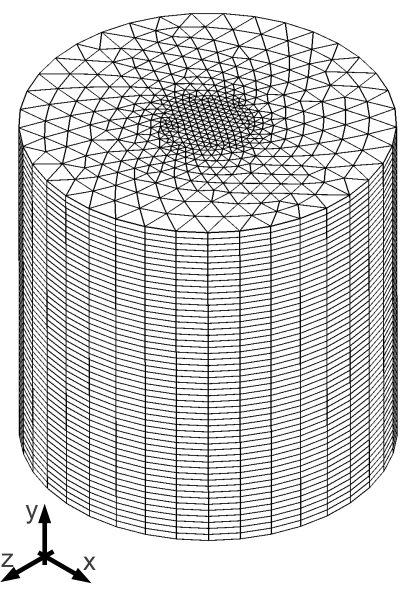

(c)

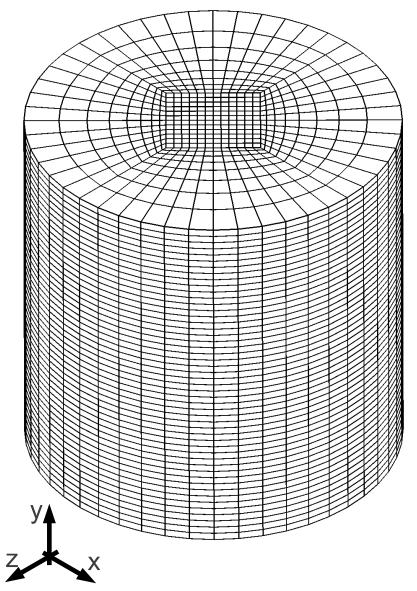

Figure 9: Three-dimensional buoyant bubbles, (a) Computational setup and initial conditions. (b) Unstructured mesh with triangular prism cells. (c) Unstructured mesh with hexahedral cells.

confinement on the terminal velocity of the bubble will be given by $U_{T} / U_{T}^{\infty} \approx$ 0.984, hence, wall effect is minimized, while the bubble has enough approach distance to reach a terminal velocity before its impact with the top wall. Regarding the applied boundary conditions, a no-slip condition is imposed at the top wall $\left(y=H_{\Omega}\right)$ and bottom wall $(y=0)$, whereas a Neumann condition is used at the lateral boundary, $r=0.5 D_{\Omega}$ (see Fig. 9a).

An unstructured mesh formed by triangular prism cells, as described in Fig. 9b, is selected unless otherwise stated. A second mesh with hexahedral cells is also used for the sake of comparison, as is illustrated in 9c. These meshes were generated by a constant step extrusion of a two-dimensional unstructured grid along the symmetry axis of the cylindrical domain, being 


\begin{tabular}{llllll}
\hline Mesh name & Cells & Cells/planes & Planes & Cell geometry & $h$ \\
\hline M1 & $3.29 \times 10^{5}$ & 2747 & 120 & Triangular prism & $d / 15$ \\
M2 & $7.98 \times 10^{5}$ & 4986 & 160 & Triangular prism & $d / 20$ \\
M3 & $2.92 \times 10^{6}$ & 12163 & 240 & Triangular prism & $d / 30$ \\
M4 & $6.75 \times 10^{6}$ & 21096 & 320 & Triangular prism & $d / 40$ \\
M5 & $1.29 \times 10^{6}$ & 5373 & 240 & Hexahedral & $d / 30$ \\
\hline
\end{tabular}

Table 6: Mesh parameters used in simulations of 3D rising bubbles. Here $d$ is the initial bubble diameter. Meshes $M_{1}, M_{2}, M_{3}$ and $M_{4}$ are illustrated in Fig. 9b, and mesh $M_{5}$ in Fig. 9c.

the step size $H_{\Omega} / N_{\text {planes }}$, where $N_{\text {planes }}$ is the number of planes in which the vertical axis is divided (see Fig. 9b and 9c). The mesh was concentrated around the symmetry axis of the cylinder, where a uniform grid size $(h)$ was fixed in order to maximize the bubble resolution. The mesh size grows exponentially to the border, where it reaches a maximum size (see Fig. 9b and $9 \mathrm{c})$.

Following the work of [9], the relevant physical quantities for the present test case are determined by the Eötvös number $(E o)$, Morton number $(M)$, Reynolds number $(R e)$, density ratio $\left(\eta_{\rho}\right)$ and viscosity ratio $\left(\eta_{\mu}\right)$ which are defined as follows:

$$
E o=\frac{g d^{2} \Delta \rho}{\sigma} \quad M=\frac{g \mu_{1}^{4} \Delta \rho}{\rho_{1}^{2} \sigma^{3}} \quad R e=\frac{\rho_{1} U_{T} d}{\mu_{1}} \quad \eta_{\rho}=\frac{\rho_{1}}{\rho_{2}} \quad \eta_{\mu}=\frac{\mu_{1}}{\mu_{2}}
$$

where $U_{T}=\int_{\Omega_{2}} v_{y} \phi d V / \int_{\Omega_{2}} d V$ is the terminal velocity of the bubble, $\Delta \rho=$ $\rho_{1}-\rho_{2}$ specifies the density difference between the fluid phases, the subscript 1 refers to the heavier fluid and the subscript 2 to the lighter fluid. Furthermore, in order to get a quantitative measure of bubble shape, the sphericity 


\begin{tabular}{llllll}
\hline Mesh & Grid size $(h)$ & $R e$ & $\varepsilon_{r}^{R e}$ & sphericity $(\zeta)$ & $\varepsilon_{r}^{\zeta}$ \\
\hline$M_{1}$ & $d / 15$ & 7.04104 & $0.341 \%$ & 0.8160 & $1.18 \%$ \\
$M_{2}$ & $d / 20$ & 7.04088 & $0.339 \%$ & 0.8125 & $0.74 \%$ \\
$M_{3}$ & $d / 30$ & 7.02213 & $0.017 \%$ & 0.8099 & $0.42 \%$ \\
$M_{4}$ & $d / 40$ & 7.01711 & - & 0.8065 & - \\
$M_{5}$ & $d / 30$ & 7.02801 & - & 0.8085 & - \\
\hline
\end{tabular}

Table 7: Grid convergence and effect of the cell geometry, for $E o=116, M=41.1$, $\eta_{\rho}=100$ and $\eta_{\mu}=100$. Here $\varepsilon_{r}^{R e}=\left|R e_{h=d / 40}-R e\right| / R e, \varepsilon_{r}^{\zeta}=\left|\zeta_{h=d / 40}-\zeta\right| / \zeta$. Cell geometry is detailed in Table 6.

is defined as

$$
\zeta=\frac{\pi d^{2}}{\int_{\Omega}\|\nabla f\| d V}
$$

\subsubsection{Grid convergence and cell geometry}

Fig. 10 and Table 7 show the effect of mesh refinement on the convergence of terminal Reynolds number and sphericity, for the case $E_{o}=116$, $M=41.1, \eta_{\rho}=100$ and $\eta_{\mu}=100$. There is a very slight difference in results obtained with meshes $M_{3}$ and $M_{4}$, which correspond to $h=d / 30$ and $h=d / 40$ respectively, therefore mesh $M_{3}$ will be used in discussion of numerical results unless otherwise stated. Moreover, it is observed that estimated errors illustrated in Table 7, are reduced with grid refinement. Regarding the influence of cell geometry on numerical results, Fig. Fig. 10 and Table 7 show a very close agreement in the calculated Reynolds number and sphericity, using the meshes $M_{3}$ and $M_{5}$, which are formed by triangular prisms and hexahedral volumes respectively. 
(a)

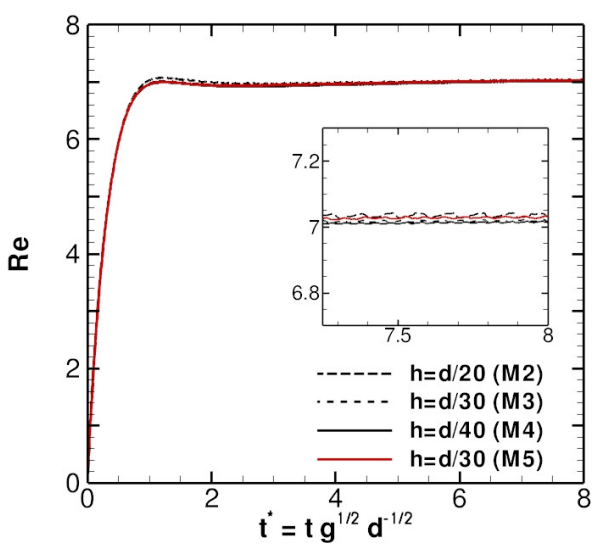

(b)

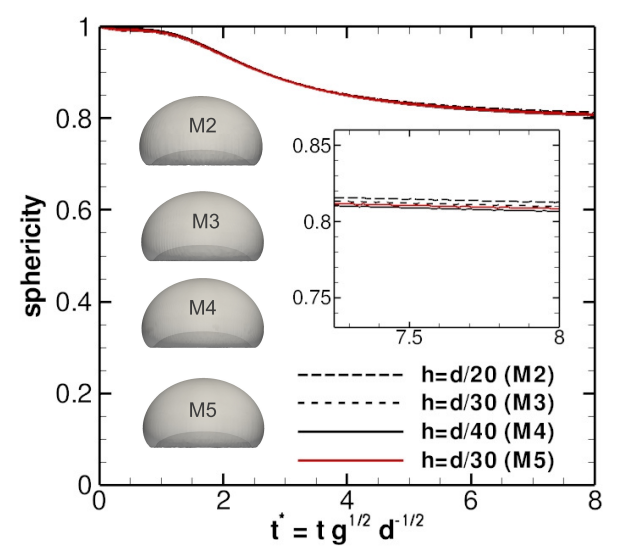

Figure 10: Grid convergence, $E o=116, M=41.1, \eta_{\mu}=100$ and $\eta_{\rho}=100$. Mesh description $\left(M_{1} \ldots M_{5}\right)$ in Table 6. (a) Reynolds number. (b) Sphericity (bubble shape).

\subsubsection{Effect of convective schemes}

Numerical simulations have been performed in order to study the influence of the convective scheme used to discretize momentum Eq. 6, on the terminal Reynolds number and bubble sphericity. Following the work of [1], the finite-volume discretization of the convective term of Eq. 6 is based on the use of flux limiters [44], $L(\theta)$, defined as

$$
L(\theta) \equiv \begin{cases}1 & \text { Central difference limiter (CD), } \\ \max \{0, \min \{2 \theta, 1\}, \min \{2, \theta\}\} & \text { TVD Superbee limiter, } \\ \frac{\theta+|\theta|}{1+|\theta|} & \text { TVD Van-Leer limiter, } \\ 0 & \text { First-order upwind limiter. }\end{cases}
$$


(a)

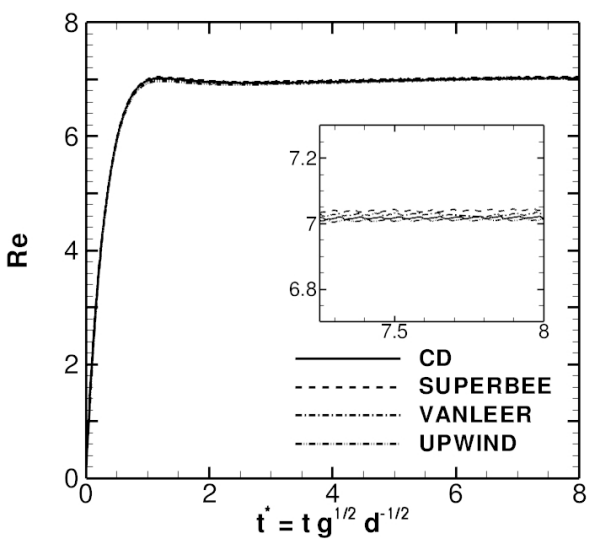

(b)

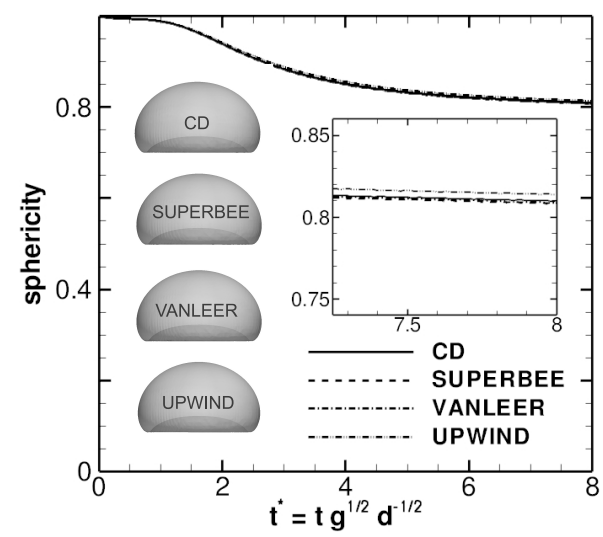

Figure 11: Effect of the convective scheme used to discretize momentum Eq. 6. Parameters $E o=116, M=41.1, \eta_{\mu}=100$ and $\eta_{\rho}=100$. Mesh $M_{3}$ with $h=d / 30$, details in Table 6. (a) Reynolds number. (b) Sphericity (bubble shape).

where $\theta$ is a monitor variable defined as the upwind ratio of consecutive gradients of the velocity components. The reader is referred to [1] for technical details on the application of flux limiters to discretize the convective term on unstructured grids. Regarding the numerical results, Fig. 11 and Table 8 show that the use of different flux limiters lead to similar results for terminal Reynolds number and sphericity, therefore a $C D$ limiter will be used on the discussion of numerical results unless otherwise stated. However, a TVD-Superbee limiter is also employed in order to avoid numerical instabilities, for instance, in flows that include high Reynolds numbers, $\eta_{\rho} \geqslant 10^{3}$ and $\eta_{\mu} \geqslant 10^{3}$, or simulations with topology changes. 


\begin{tabular}{lll}
\hline Flux limiter & $R e$ & sphericity $(\zeta)$ \\
\hline CD & 7.02213 & 0.8099 \\
Superbee & 7.04084 & 0.8086 \\
Van Leer & 7.03323 & 0.8089 \\
Upwind & 7.01109 & 0.8139 \\
\hline
\end{tabular}

Table 8: Effect of the convective scheme used to discretize momentum Eq. 6, on the terminal Reynolds number and sphericity, for $E o=116, M=41.1, \eta_{\rho}=100$ and $\eta_{\mu}=100$. Mesh $M_{3}$ with $h=d / 30$.

\subsubsection{Bubble shapes and terminal velocity}

For the sake of comparison, experimental results found in $[4,21,9]$ and numerical results reported by [22] are used as reference. Fig. 12a shows the effect of Morton number on the bubble dynamics, given $E o=116, \eta_{\rho}=100$, $\eta_{\mu}=100$ and Morton number varying from 5.51 up to 848. It is observed that Re tends to a steady state value for all $M$, however, as the Morton number increases the overshoot on $R e$ is more pronounced, indicating that the bubble motion has a tendency to reduce its stability. Particularly, the characteristic overshoot of the instantaneous Reynolds number after the bubbles start to ascend is well represented in the cases with $M>5.51$. Regarding the mass conservation error of the bubble phase, Fig. 12b proves the satisfaction of this requirement, where a maximum error of $O\left(10^{-5}\right)$ is observed. Here, the instantaneous mass is calculated and compared with the initial mass, then mass conservation error is calculated by the expression $M_{r}=\mid M(t)-$ $M(0) \mid / M(0)$ with $M(t)=\int_{\Omega} f d V$.

Fig. 13 illustrates a qualitative comparison of the calculated bubble shapes against experimental images reported by [4], for a wide range of Eo and $M$, with $\eta_{\rho}=100$ and $\eta_{\mu}=100$. Moreover, Table 9 shows a quanti- 
(a)

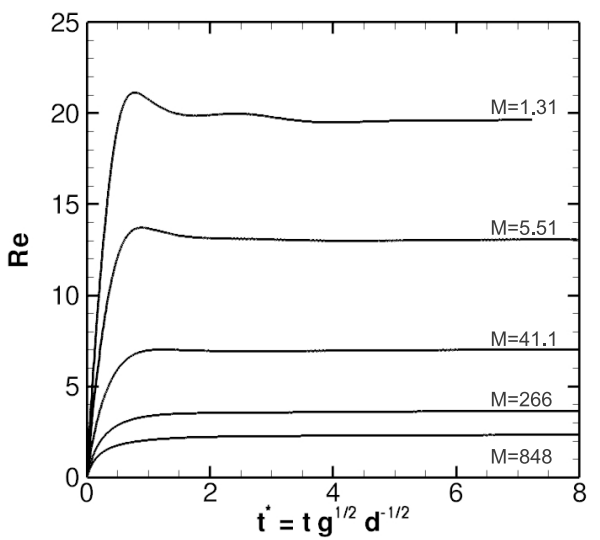

(b)

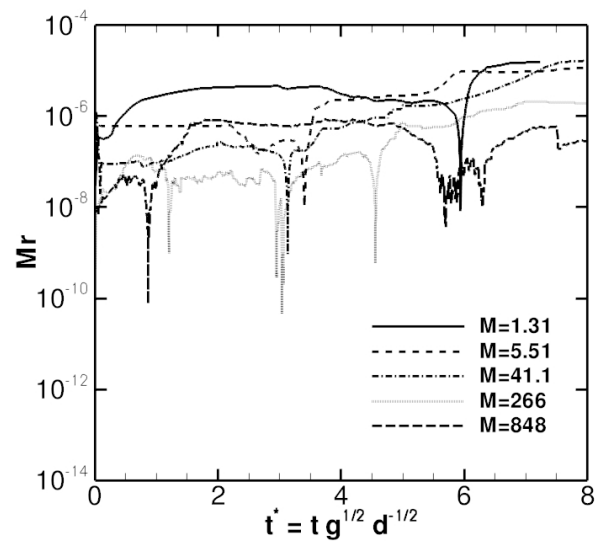

Figure 12: $E o=116,1.31 \leq M \leq 848, \eta_{\mu}=100$ and $\eta_{\rho}=100$. (a) Time evolution of the Reynolds number. (b) Mass conservation error $\left(M_{r}=|M(t)-M(0)| / M(0)\right)$. Here $M(t)=\int_{\Omega} f(\mathbf{x}, t) d V$. The maximun mass conservation error is $O\left(10^{-5}\right)$.

tative comparison of terminal Reynolds numbers calculated by the present VOF/LS method against experimental data of [4] and numerical results reported by [22]. In addition Table 10 presents a comparison of VOF/LS results against experimental data taken from the Grace diagram [9], for $E o=10$, $10^{-3}<M<10, \eta_{\rho}=100$ and $\eta_{\mu}=100$. Further details of the bubble dynamics, including time evolution of Reynolds number, sphericity, bubble shapes and wake patterns are illustrated in Figs. 14-19. Given the aforementioned results, it is observed that present simulations are in close agreement with previous results from the literature, moreover, numerical stability and accuracy of present VOF/LS method has been proved for a wide range of dimensionless parameters.

As further validation for high density and viscosity ratios, a rising bubble 


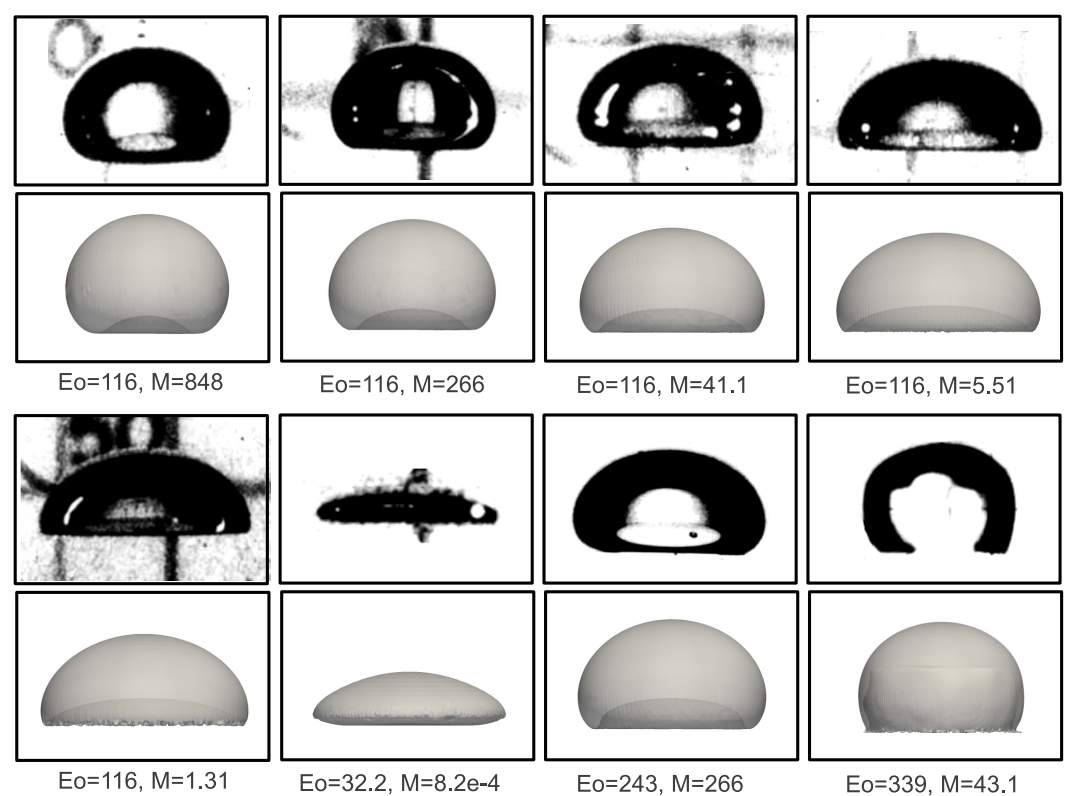

Figure 13: Terminal bubbles shapes reported in experiments of [4] (top rows) and computations performed by the present VOF/LS method (bottom rows). Simulations were performed using $\eta_{\rho}=100$ and $\eta_{\mu}=100$.

\begin{tabular}{llllll}
\hline & & \multicolumn{3}{c}{$R e$} & \\
\cline { 3 - 5 } Eo & $M$ & {$[4]$} & {$[22]$} & Present & Mesh \\
\hline 116 & 848 & 2.47 & 2.317 & 2.33 & $M_{3}$ \\
116 & 266 & 3.57 & 3.621 & 3.65 & $M_{3}$ \\
116 & 41.1 & 7.16 & 7.0 & 7.02 & $M_{3}$ \\
116 & 5.51 & 13.3 & 13.17 & 13.06 & $M_{3}$ \\
116 & 1.31 & 20.4 & 19.88 & 19.65 & $M_{4}$ \\
32.2 & $8.2 \times 10^{-4}$ & 55.3 & 52.96 & 52.84 & $M_{3}$ \\
243 & 266 & 7.77 & 8.397 & 7.84 & $M_{3}$ \\
339 & 43.1 & 18.3 & 17.91 & 17.64 & $M_{4}$ \\
\hline
\end{tabular}

Table 9: Present computations for $E o=116,1.31 \leq M \leq 848, \eta_{\rho}=100$ and $\eta_{\mu}=100$, compared against experimental results from [4] and numerical results from [22]. 


\begin{tabular}{lllll}
\hline & & \multicolumn{3}{c}{$R e$} \\
\cline { 3 - 4 } Eo & $M$ & {$[9]$} & Present & Mesh \\
\hline 10 & $10^{-3}$ & 23.6 & 23.53 & $M_{3}$ \\
10 & $10^{-2}$ & 11.7 & 11.37 & $M_{3}$ \\
10 & $10^{-1}$ & 4.9 & 4.92 & $M_{3}$ \\
10 & 1 & 1.7 & 1.95 & $M_{3}$ \\
10 & 10 & 0.6 & 0.70 & $M_{3}$ \\
\hline
\end{tabular}

Table 10: Present computations for $E_{o}=10,10^{-3} \leq M \leq 10, \eta_{\rho}=100$ and $\eta_{\mu}=100$, compared against experimental results taken from the Grace diagram [9].

(a)

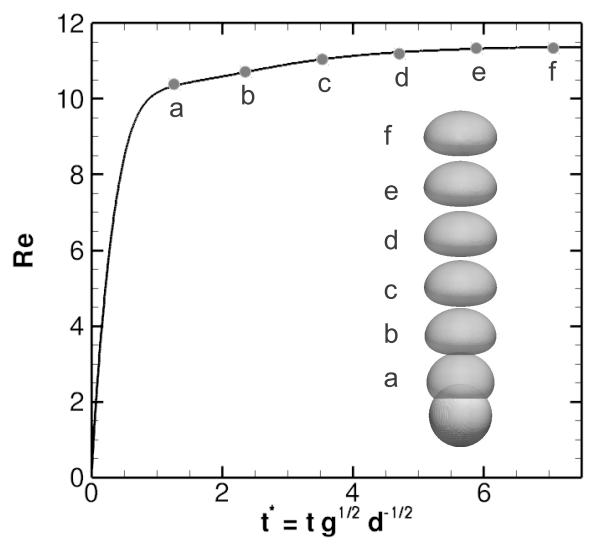

(b)

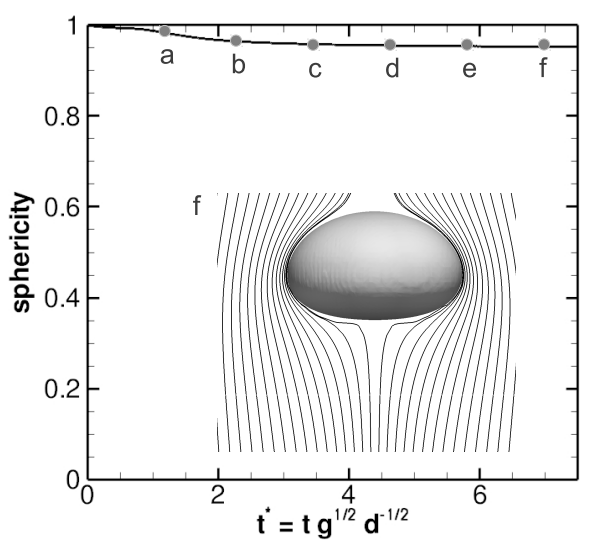

Figure 14: Buoyant bubble for $E o=10, M=1 \times 10^{-2}, \eta_{\rho}=100$ and $\eta_{\mu}=100$. Mesh $M_{3}$. (a) Reynolds number and bubble shape evolution. (b) Sphericity and streamlines 
(a)

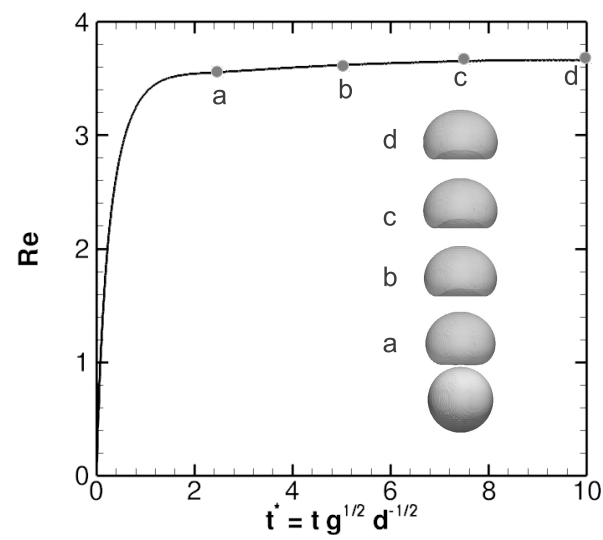

(b)

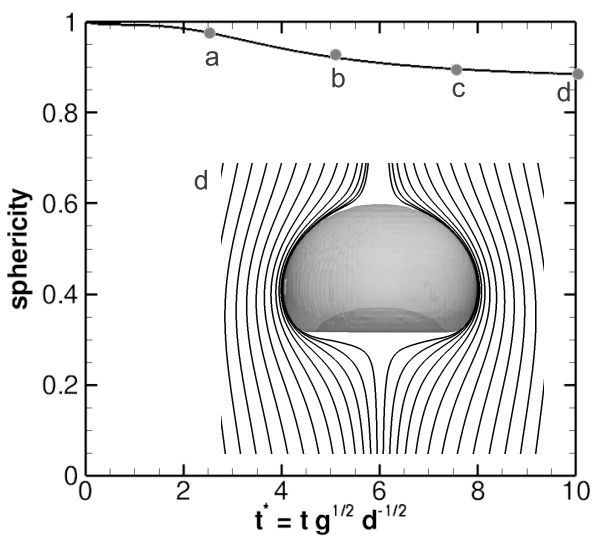

Figure 15: Buoyant bubble for $E o=116, M=266, \eta_{\rho}=100$ and $\eta_{\mu}=100$. Mesh $M_{3}$. (a) Reynolds number and bubble shape evolution. (b) Sphericity and streamlines

(a)

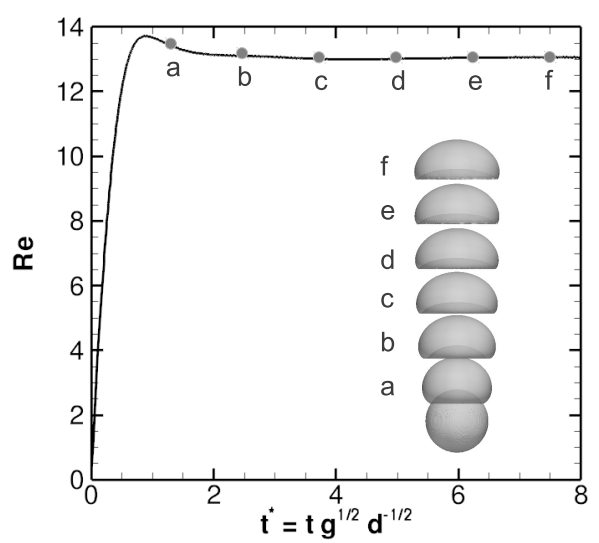

(b)

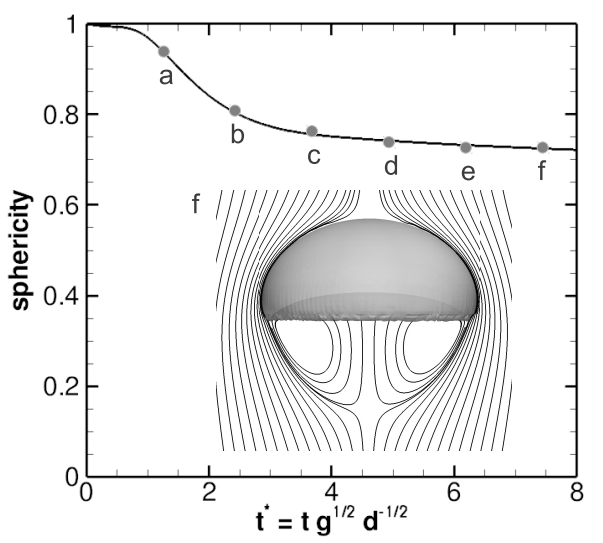

Figure 16: Buoyant bubble for $E o=116, M=5.51, \eta_{\rho}=100$ and $\eta_{\mu}=100$. Mesh $M_{3}$. (a) Reynolds number and bubble shape evolution. (b) Sphericity and streamlines 
(a)

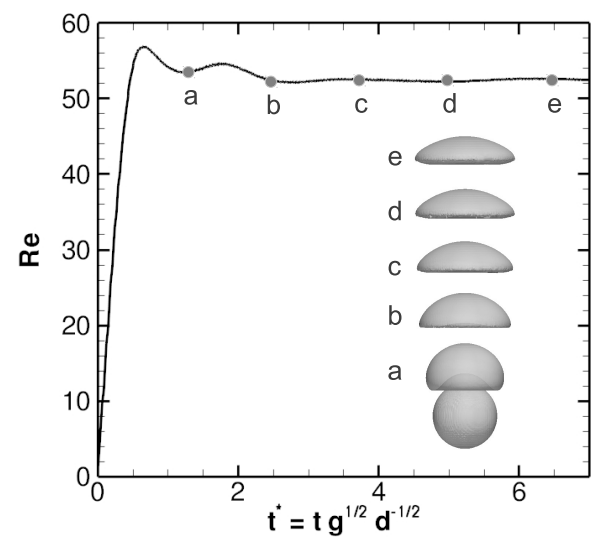

(b)

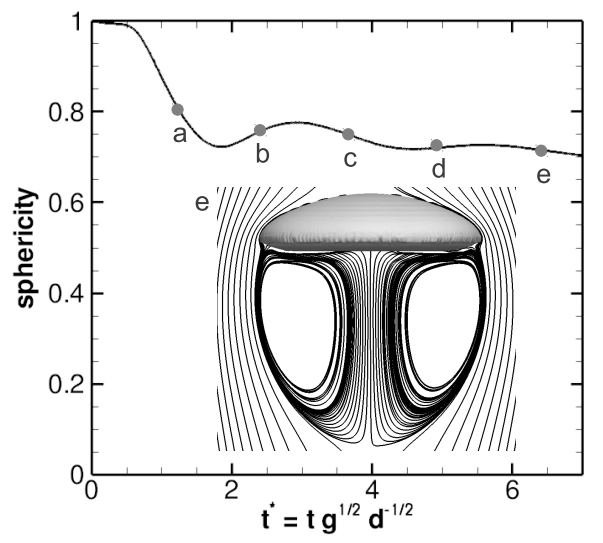

Figure 17: Buoyant bubble for $E o=32.2, M=8.2 \times 10^{-4}, \eta_{\rho}=100$ and $\eta_{\mu}=100$. Mesh $M_{3}$. (a) Reynolds number and bubble shape evolution. (b) Sphericity and streamlines

(a)

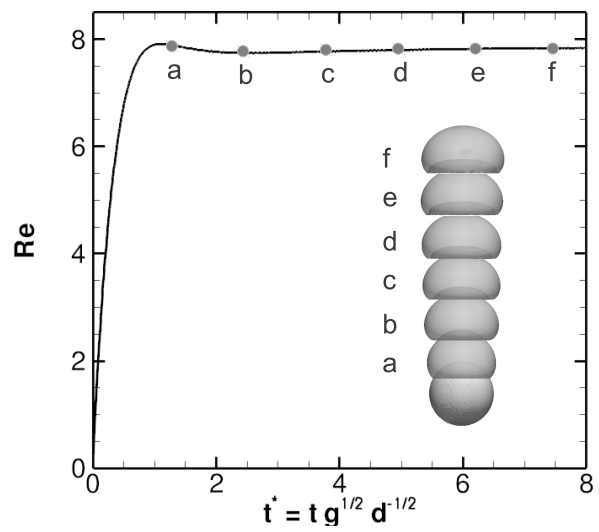

(b)

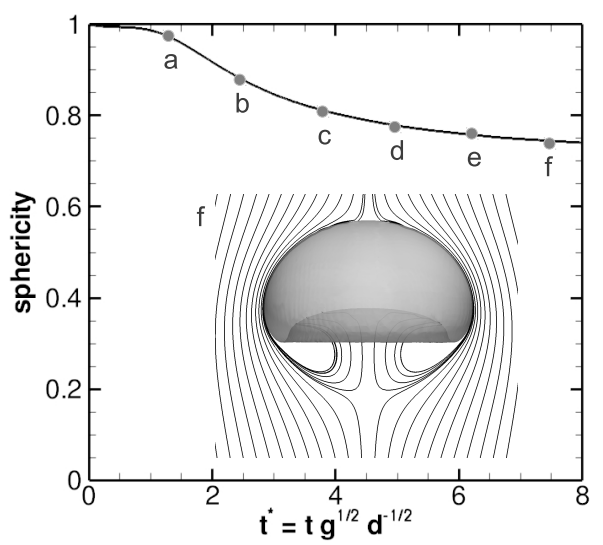

Figure 18: Buoyant bubble for $E o=243, M=266, \eta_{\rho}=100$ and $\eta_{\mu}=100$. Mesh $M_{3}$. (a) Reynolds number and bubble shape evolution. (b) Sphericity and streamlines 
(a)

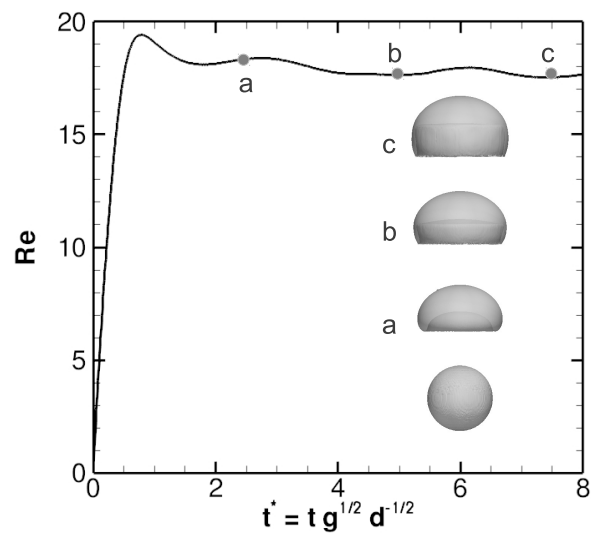

(b)

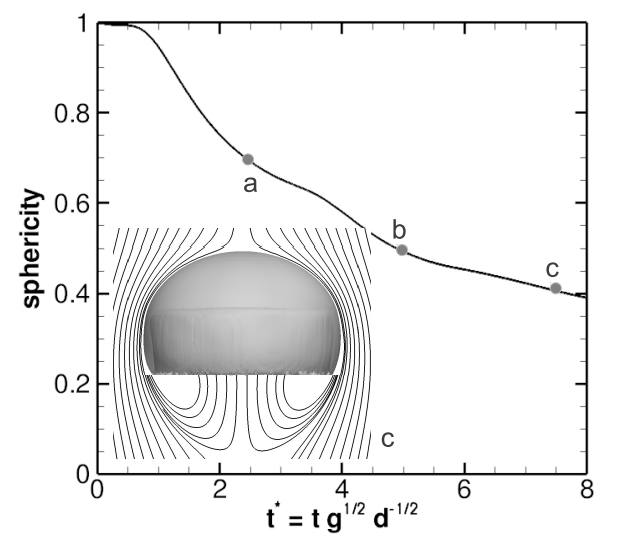

Figure 19: Buoyant bubble for $E o=339, M=43.1, \eta_{\rho}=100$ and $\eta_{\mu}=100$. Mesh $M_{4}$. (a) Reynolds number and bubble shape evolution. (b) Sphericity and streamlines

(a)

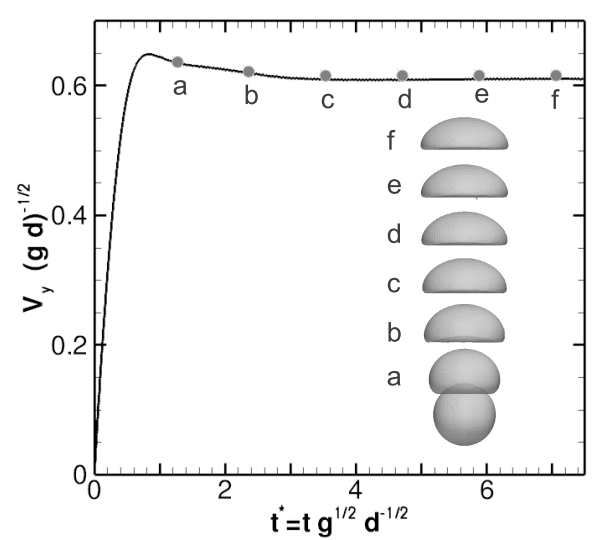

(b)

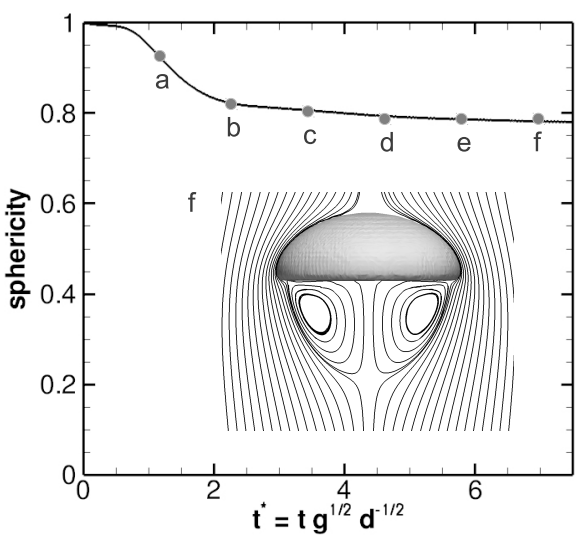

Figure 20: Buoyant bubble for $E_{o}=39.4, M=0.065, \eta_{\rho}=714$ and $\eta_{\mu}=6670$. Mesh $M_{3}$. (a) Dimensionless velocity, $\mathbf{v}_{b} \cdot \mathbf{e}_{y}(g d)^{-1 / 2}$. (b) Sphericity and streamlines 

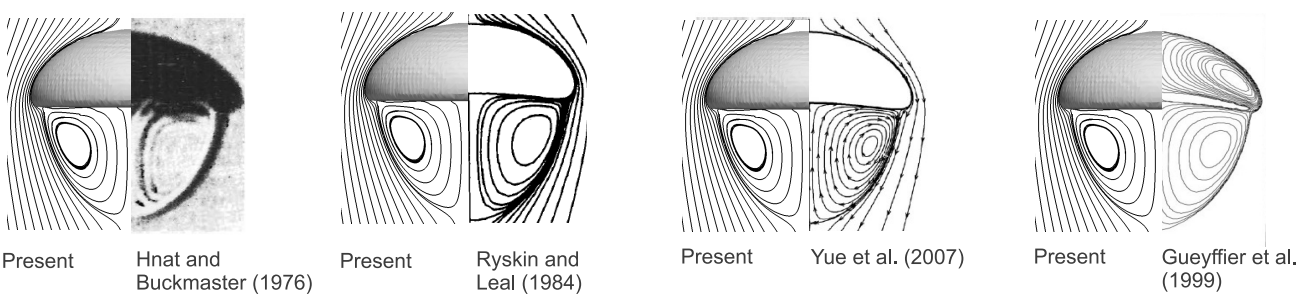

Figure 21: Comparison of wake structure reported in experiment of [21] and numerical results of the literature $[35,37,14]$, against computations using the present VOF/LS method. $E o=39.4, M=0.065, \eta_{\rho}=714$ and $\eta_{\mu}=6670$.

is simulated for dimensionless parameters $E o=39.4, M=0.065, \eta_{\rho}=714$ and $\eta_{\mu}=6670$, which corresponds to experimental conditions used in one of the cases reported by [21]. In order to avoid numerical instabilities in this case with high density and viscosity ratios, a TVD-Superbee limiter is used to discretize the convective term of momentum Eq. 6. Fig. 20a shows that bubble is deformed until get a spherical cap shape at steady state, moreover the terminal velocity calculated by $\mathrm{VOF} / \mathrm{LS}$ method is $U_{T, \text { num }}^{*}=$ $\mathbf{v} \cdot \mathbf{e}_{z}(g d)^{-1 / 2}=0.6110$, whereas [21] reported a terminal velocity of $U_{T, \exp }=$ $0.215 \mathrm{~m} / \mathrm{s}$ or equivalently $U_{T, \exp }^{*}=0.6226$. Therefore, a slight difference of $1.8 \%$ is observed between present results and experiment reported by [21]. Fig. 21 illustrates a comparison of the bubble shape and wake structure based on the present VOF/LS method, against experiment of [21], and other interface capturing methods, including the body-fitted method [35], ALE approach [37] and VOF approach [14]. Indeed, present result is in close agreement with experiment of [21], and other numerical studies from the literature. 


\subsubsection{Drag coefficient and comparison with empirical correlation}

In this section the numerical drag coefficient $\left(C_{D}\right)$ is compared against experimental correlations and theoretical predictions from the literature. The drag coefficient can be obtained from a simulation where a bubble is released in an initially quiescent liquid. After to apply a force balance in the vertical direction at steady state, $C_{D}$ can be expressed in terms of the terminal rise velocity $U_{T}$ :

$$
C_{D}=\frac{4\left(\rho_{l}-\rho_{g}\right)\|\mathbf{g}\| d}{3 \rho_{l} U_{T}^{2}}
$$

[4] proposed an experimental correlation $C_{D}=f(R e)$ for fluids with Morton number $M>4 \times 10^{-3}$ :

$$
C_{D}=\left((2.67)^{0.9}+(16 / R e)^{0.9}\right)^{1 / 0.9} \quad M>4 \times 10^{-3}
$$

Moreover, [23] reports a drag coefficient based on theoretical predictions:

$$
C_{D}=0.445\left(6+\frac{32}{R e}\right)
$$

[16] and [39] generalize the Stokes result for the viscous drag force on a solid sphere, to fluid particles of arbitrary and finite internal viscosity, inferring the drag coefficient at very low Reynolds numbers:

$$
C_{D}=\frac{8}{R e} \frac{2+3 \eta_{\mu}^{-1}}{1+\eta_{\mu}^{-1}} \quad R e \ll 1
$$


(a)

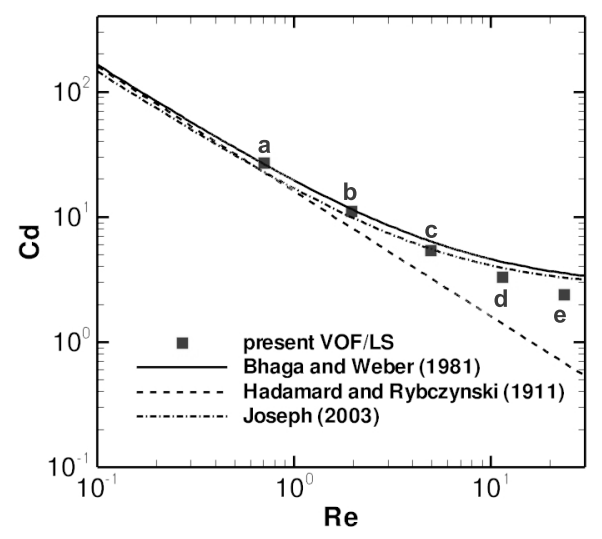

(b)

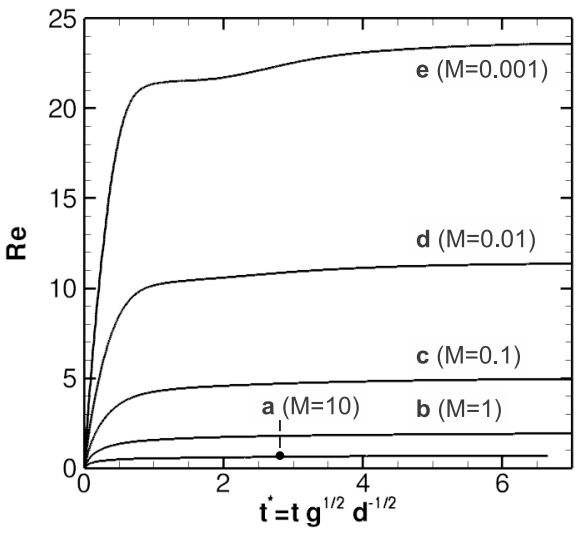

(c)

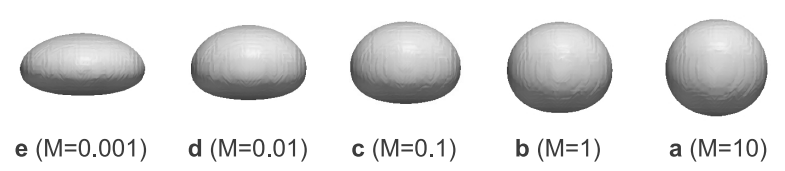

Figure 22: $\quad E o=10, \eta_{\rho}=100, \eta_{\mu}=100,10^{-3} \leq M \leq 10$. (a) Drag coefficient $\left(C_{d}\right)$ versus Reynolds number $(R e)$. (b) Reynolds number versus dimensionless time $t^{*}=t g^{1 / 2} d^{-1 / 2}$. (c) Terminal bubbles shapes.

Fig. 22a illustrates the drag coefficient versus Reynolds number for dimensionless parameters given by $E_{O}=10,10^{-3} \leq M \leq 10^{2}, \eta_{\rho}=100$ and $\eta_{\mu}=100$, while Figure 22b shows the time evolution of Reynolds number for the aforementioned cases. Here it is observed that if $M$ decreases then the bubble deformation increases, as shown in Fig. 22c, therefore the spherical bubble approximation given by Eq. 43 is valid for only high $M$. Moreover, these numerical predictions for $C_{D}$ are in good agreement with correlations given by Eq. 41 and Eq. 42 .

Now, the numerical results presented in Table 9 and Table 10, for $E o=$ 


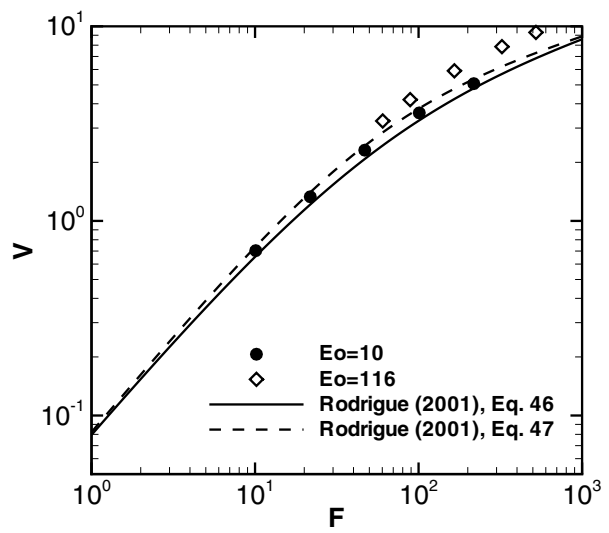

Figure 23: Velocity number $(V)$ as a function of the flow number $(F)$. Correlations are taken from [34].

116 and $E o=10$ respectively, are used to evaluate the flow number $(F)$ and the velocity number $(V)$, defined as:

$$
\begin{aligned}
& F=g\left(\frac{d^{8} \rho_{l}^{5}}{\sigma \mu_{l}^{4}}\right)^{1 / 3} \\
& V=U_{t}\left(\frac{d^{2} \rho_{l}^{2}}{\sigma \mu_{l}}\right)^{1 / 3}
\end{aligned}
$$

Numerical results are compared to the empirical correlations reported by [34]:

$$
V=\frac{F}{12\left(1+0.049 F^{3 / 4}\right)}
$$




$$
V=\frac{F}{12(1+0.0185 F)^{3 / 4}}
$$

Fig. 23 shows that numerical predictions are in good agreement with Eq. 46 and Eq. 47.

\subsection{Coaxial coalescence of two bubbles}

In this section the co-axial coalescence of two bubbles is simulated. The spatial domain is the same that the previous test case, moreover it is divided by triangular prisms cells according to the mesh $M_{3}$ described in Table 6 . As initial condition two spherical bubbles of diameter $d$ are aligned along to the $z$-axis of the cylindrical domain (see Fig. 9), separated by a centroidcentroid vertical distance of $1.5 \mathrm{~d}$. Both bubbles and their surrounding liquid are initially quiescent, moreover fluid properties are given by the nondimensional parameters $E_{O}=16, M=2 \times 10^{-4}, \eta_{\rho}=100$ and $\eta_{\mu}=100$, which corresponds to the zone between skirted and spherical cap regimes according to the Grace Diagram [9]. Free-slip boundary condition is used at the lateral wall and no-slip boundary condition at the top and bottom boundaries. Finally, in order to avoid numerical instabilities, a TVD-Superbee flux limiter has been used to discretize the convective term of momentum equation.

Fig. 24a shows the time evolution of Reynolds number, whereas Fig. 24b illustrates the evolution of the interfacial surface, and stream-lines around the merged bubble. In addition, snapshots of topological changes during the 
(a)

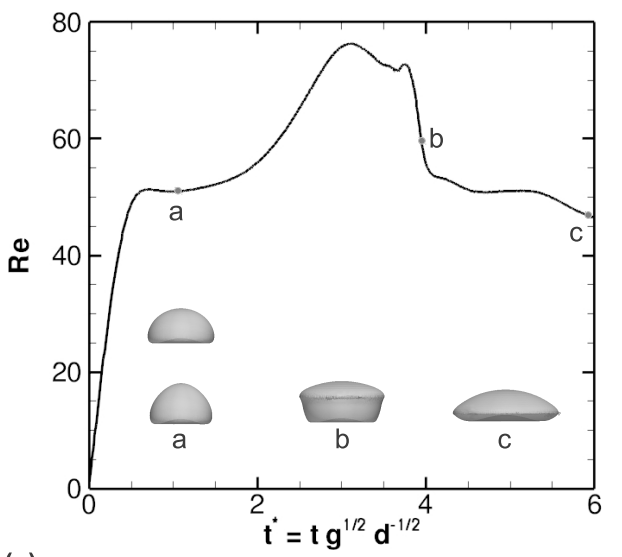

(c)
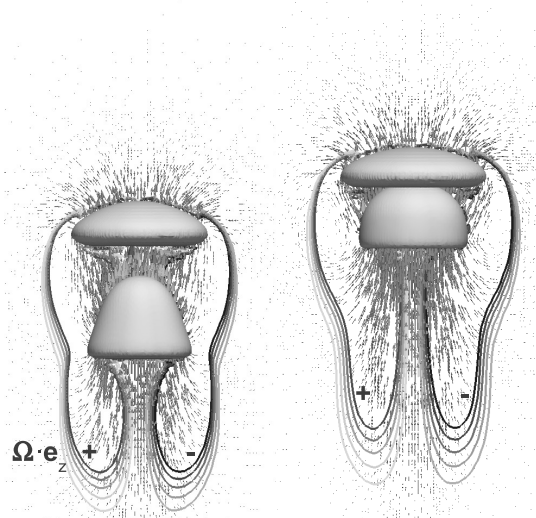

(b)

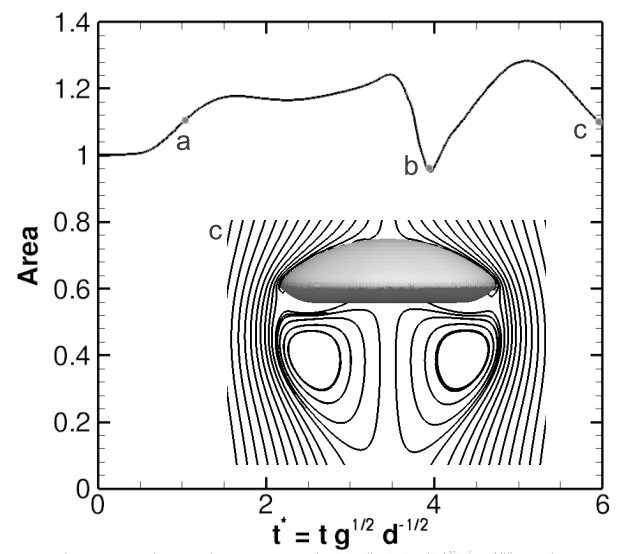

Figure 24: Coaxial coalescence of two bubbles, $E o=16, M=2 \times 10^{-4}, \eta_{\rho}=100$ and $\eta_{\mu}=100$. Mesh $M_{3}$. (a) Reynolds number, $\operatorname{Re}(t)=\rho_{c} \mu_{c}^{-1} d_{b} \int_{\Omega} f(\mathbf{x}, t) \mathbf{v}(\mathbf{x}, t) \cdot \mathbf{e}_{y} d V$. (b) Dimensionless interfacial area $A(t) / A(0)$ with $A(t)=\int_{\Omega}\|\nabla f(\mathbf{x}, t)\| d V$, and streamlines. (c) Velocity vectors and vorticity contours $\Omega \cdot \mathbf{e}_{z}$ on the plane $x-y$ at $z=0$, for $t^{*}=$ $\{2.77,3.56,4.36,5.14\}$. 
(a)

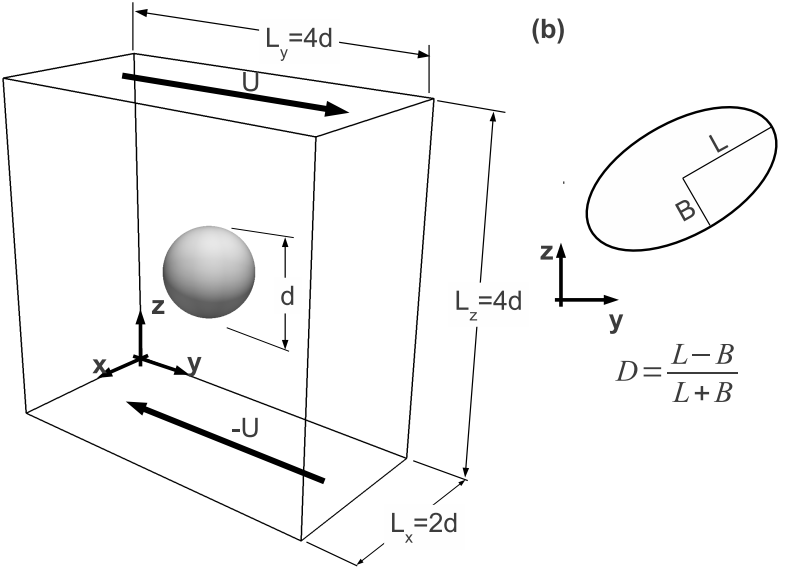

(c)

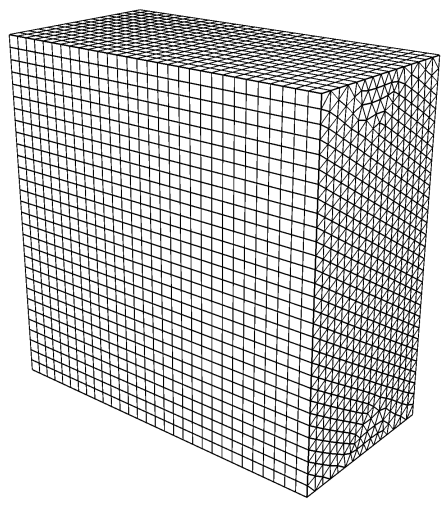

Figure 25: (a) Computational setup and initial condition. (b) Droplet deformation parameter, D. (c) Mesh configuration, triangular prism cells.

coalescence process are illustrated in Fig. 24c. As the bubbles rise, a liquid jet is formed behind the leading bubble, which induces a severe deformation in vertical direction of the following bubble. Then, once the two bubbles are approaching, the trailing bubble accelerates because the suction by the top bubble. As time progresses, the two bubbles start to touch, leaving a mushroom-like structure. Finally, the thin liquid film between bubbles is squeezed out and ruptured, completing the coalescence process. Numerical predictions match fairly well in terms of bubble shapes with experimental results reported by [6]. Moreover, present results are consistent with VOF simulation from [48] and level-set simulations reported by [1].

\subsection{Deformation of a droplet in a shear flow}

A spherical drop of diameter $d$ is located at the center of a computational domain $x \in[0,8], y \in[0,4], z=\in[0,8]$, without effect of gravity force, 
(a)
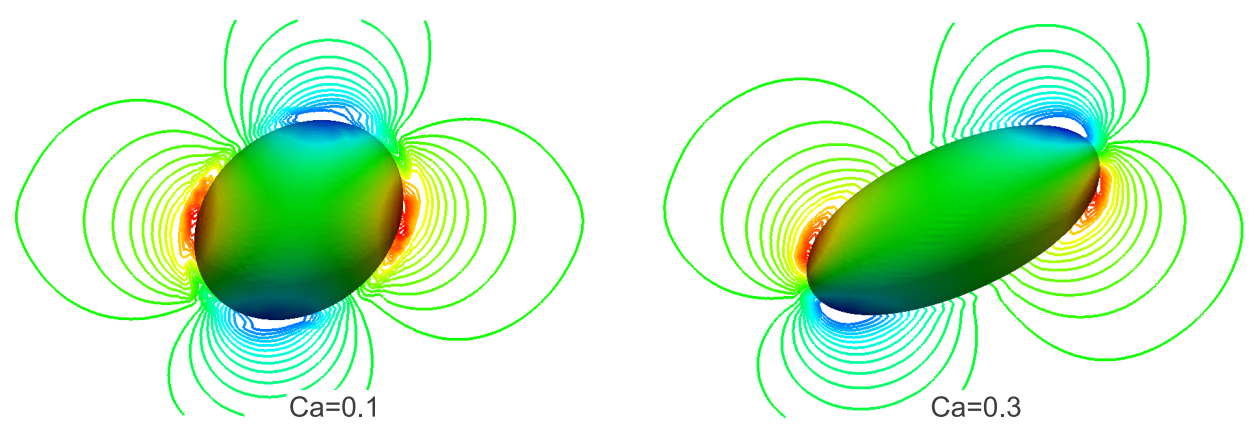

(b)

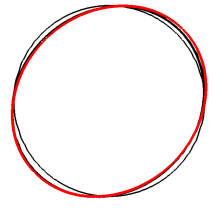

$\mathrm{Ca}=0.05$

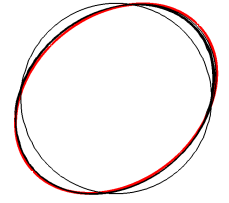

$\mathrm{Ca}=0.1$

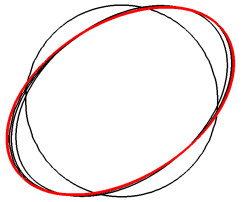

$\mathrm{Ca}=0.2$

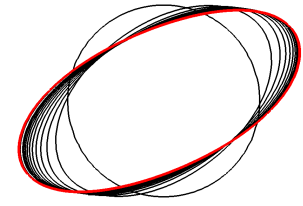

$\mathrm{Ca}=0.3$

Figure 26: (a) Vorticity contours $\left(\mathbf{e}_{x} \cdot \nabla \times \mathbf{v}\right)$ on the droplet surface and on the plane $z-y$ at $x=0$. (b) Time evolution of droplet shapes on the plane $z-y$ at $x=0$. Here $\eta_{\rho}=1, \eta_{\mu}=1, R e=0.1,0.05 \leqslant C a \leqslant 0.3$. Red line used for steady state shape.

(a)

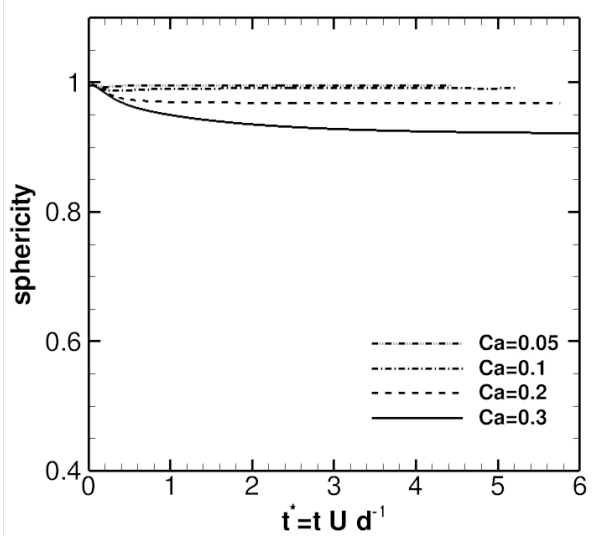

(b)

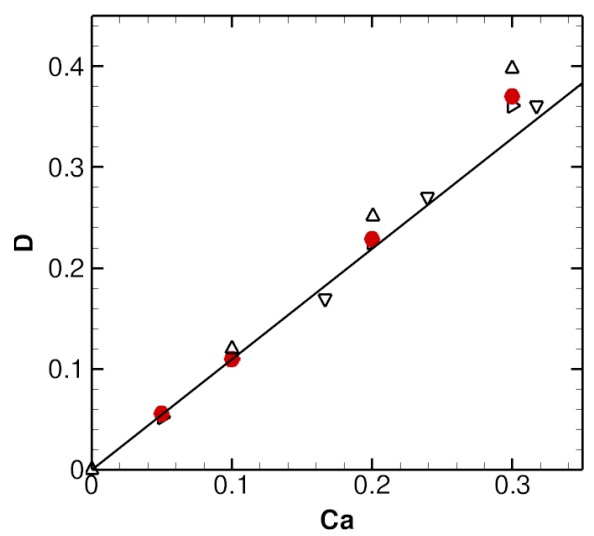

Figure 27: (a) Droplet sphericity versus time. (b) Capillary number $C a$ versus Taylor deformation parameter $D$. Present VOF/LS method (red symbol); $\triangleright$ VOF [27]; - theory [45]; $\triangle$ Lattice-Boltzmann method [54]; $\nabla$ boundary integral method [52]; 
as shown in Fig. 25a. The opposite velocities $\pm U$ are imposed on the top and bottom walls, periodic boundary condition is applied in $x$ direction and Neumann boundary condition in $y$ direction. The initial condition at time $t=0$ is a drop with spherical form and the initial velocity field is linear inside the computational domain. Computations have been performed using an unstructured mesh formed by $1.94 \times 10^{6}$ triangular prism cells with grid size $h=d / 30$, as is illustrated in Fig. 25c. This mesh was generated by a constant step extrusion of a two-dimensional triangular grid along the $y$-axis, being $h$ the step size.

The deformation behaviour of the droplet is determined by the Reynolds number $(R e)$ and the capillary number $(C a)$. The viscosity is given by the Reynolds number

$$
R e \equiv \frac{\rho_{c} \dot{\gamma} d^{2}}{4 \mu_{c}}
$$

where the shear rate is defined by $\dot{\gamma}=2 U / L_{z}$. The capillary number is given by

$$
C a \equiv \frac{\dot{\gamma} \mu_{d} d}{2 \sigma}
$$

This dimensionless parameter is a measure of the relative effect of the shear stress versus the surface tension across the fluid-fluid interface. The Reynolds number used in these test cases is $R e=0.1$, while the capillary number is in the range $0.05 \leqslant C a \leqslant 0.3$. The same viscosity and density are specified for 
both drop fluid and continuous fluid, thus $\eta_{\rho}=\rho_{c} / \rho_{d}=1$ and $\eta_{\mu}=\mu_{c} / \mu_{d}=$ 1, where the sub-index $c$ is used for the continuous fluid and $d$ the droplet fluid.

The droplet shapes at steady state and some vorticity distributions are illustrated in Fig. 26. The interface becomes ellipsoidal and its deformation and rotation are larger as the capillary number increases. A theoretical solution was derived by [45] to predict small distortions of the droplets from the spherical form at slow speeds, on the hypothesis of Stokes flow. This result show that the droplet is distorted into an ellipse where the deformation parameter given by $D=(L-B)(L+B)^{-1}$ (see Fig. 25b) is linearly changed with the capillary number (Eq. 49). Here $L$ and $B$ denote the semi-major and semi-minor axes of the ellipse, as is illustrated in Fig. 25b. Fig. 27a shows the time evolution of droplet sphericity for $0.05 \leq C a \leq 0.3$, whereas in Fig. 27b are plotted the Taylor deformation parameter $(D)$ versus capillary number $(\mathrm{Ca})$. Here, it is observed a close agreement between present computations using VOF/LS method, against previous results from the literature [45, 27, 54, 52]. Moreover, for small capillary numbers the shape in steady state is close to the theoretical predictions of [45], while the theory underestimates the droplet deformation parameter for large capillary numbers, as shown Fig. $27 \mathrm{~b}$. 


\section{Conclusions}

A coupled VOF/LS method, which combines the advantages and overcomes the disadvantages of both techniques, has been proposed for computing incompressible two-phase flows on unstructured meshes. From the comparison of the present numerical simulations against experiments and numerical data from the literature, it is possible to conclude that this method is enough robust to perform high accurate computations of interfacial flows with surface tension. Moreover, an error less than $O\left(10^{-5}\right)$ in the mass conservation property of the fluid phases is achieved because a VOF-PLIC method is used for interface advection, while an accurate computation of interface curvature and surface tension is performed by means of a level-set function reconstructed from a geometrical algorithm. In addition, numerical stability of the present unstructured VOF/LS solver has been proved for a wide range of dimensionless parameters, including simulations with high density and high viscosity ratios, and interfacial flow with topological changes. Altogether, these validations demonstrate that the present VOF/LS approach and the developed code for simulating two-phase flows on collocated unstructured meshes can be used for practical applications.

\section{Acknowledgments}

This work has been financially supported by the Ministerio de Economía y Competitividad, Secretaría de Estado de Investigación, Desarrollo e Innovación, Spain (ENE2011-28699), and by Termo Fluids S.L. Néstor Balcázar 
acknowledges financial support in form of a doctoral scholarship of the Agencia Española de Cooperación Internacional para el Desarrollo (AECID), Spain. Three-dimensional simulations were carried out using computer time provided by PRACE (project 2014112666) through the MareNostrum III supercomputer based in Barcelona, Spain.

\section{References}

[1] Balcázar, N., Jofre, L., Lehmkhul, O., Castro, J., Rigola, J., 2014. A finite-volume/level-set method for simulating two-phase flows on unstructured grids. International Journal of Multiphase Flow 64, 55-72

[2] Balcázar, N., Lemhkuhl, O., Rigola, J., Oliva, A., 2015. A multiple marker level-set method for simulation of deformable fluid particles. International Journal of Multiphase Flow 74, 125-142

[3] Balcázar, N., Lemhkuhl, O., Jofre, L., Oliva, A., 2015. Level-set simulations of buoyancy-driven motion of single and multiple bubbles. International Journal of Heat and Fluid Flow 56, 91-107

[4] Bhaga, D., Weber, M.E., 1981. Bubbles in viscous liquids: shapes, wakes and velocities, J Fluid Mech 105, 61-85

[5] Brackbill, J.U., Kothe, D.B., Zemach, C., 1992. A Continuum Method for Modeling Surface Tension, J. Comput. Phys. 100, 335-354. 
[6] Brereton, G., Korotney, D., Coaxial and Oblique Coalescence of Two Rising Bubbles, in Dynamics of bubbles and Vortices Near a Free Surface, vol. 119, ASME, New York, 1991

[7] Chorin, A.J., Numerical solution of the Navier-Stokes equations. 1968. Math. Comput. 22, 745-762.

[8] Chang, Y.C., Hou, T.Y., Merriman, B., Osher, S., A level-set formulation of Eulerian interface capturing methods for incompressible twophase flows. 1996. Journal of Computational Physics 124, 462-488.

[9] Clift, R., Grace, J.R., Weber, M.E., Bubbles, Drops and Particles. Academin Press, New York, 1978.

[10] Deen, N.G., Van Sint Annaland, M., Kuipers, J.A.M. , 2009. Direct numerical simulation of complex multi-fluid flows using a combined front tracking and immersed boundary method, Chemical Engineering Science 64, 2186-2201.

[11] Engquist, B., Tornberg, A.K., Tsai, R., 2005. Discretization of Dirac delta functions in level set methods, Journal of Computational Physics $207,28-51$.

[12] Felten, F.N., Lund, T.S., 2006. Kinetic energy conservation issues associated with the collocated mesh scheme for incompressible flow, J. Comput. Phys. 215, 465-484. 
[13] Gottlieb, S., Shu, C., 1998. Total Variation Dimishing Runge-Kutta Schemes, Mathematics of Computations 67, 73-85.

[14] Gueyffier, D., Li, J., Nadim, A., Scardovelli, R., Zaleski, S., 1999. Volume-of-fluid interface tracking with smoothed surface stress methods for three-dimensional flows, J. Comput. Phys. 152, 423-456.

[15] Haselbacher, A., Vasilyev, O., 2003. Commutative discrete filtering on unstructured grids based on least-squares techniques, J. Comput. Phys. 187, 197-211.

[16] Hadamard, J.S., 1911. Mouvement permanent lent dune sphere liquide et visqueuse dans un liquide visqueux, C. R. Acad. Sci. 152, 1735.

[17] Harmathy, T.Z., 1960. Velocity of large drops and bubbles in media of infinite or restricted extend, AIChE J. 6, 281-288.

[18] Hirt, C., Nichols, B., 1981. Volume of fluid (VOF) method for the dynamics of free boundary, J. Comput. Phys. 39, 201-225

[19] Hysing, S., Turet, S., Kuzmin, D., Parolini, N., Burman, E., Ganesan, S., Tobiska, L., 2009. Quantitative benchmark computations of two-dimensional bubble dynamics, International Journal for Numerical Methods in Fluids 60, 1259-1288.

[20] Hysing, S., 2012. Mixed element FEM level set method for numerical simulation of immiscible fluids, J. Comput. Phys. 231, 2449-2465. 
[21] Hnat, J.G., Buckmaster, J.D., 1976. Spherical cap bubbles and skirt formation, Phys. Fluids 19, 182-194

[22] Hua, J., Stene, J., Lin, P., 2008. Numerical simulation of 3D bubbles rising in viscous liquids using a front tracking method, J. Comput. Phys. $227,3358-3382$

[23] Joseph, D., 2003. Rise velocity of a spherical cap bubble, J. Fluid Mech. $488,213-223$

[24] Jofre, L., Lehmkuhl, O., Castro, J., Oliva, A., 2014. A 3-D Volume-ofFluid advection method based on cell-vertex velocities, Computers \& Fluids 94, 14-29

[25] Kothe, D.B., Rider, W.J., Mosso, S.J., Brock, J.S., 1996. Volume Tracking of Interfaces Having Surface Tension in Two and Three Dimensions, AIAA 96-0859. 25.

[26] Lehmkuhl, O., Perez-Segarra, C.D., Soria, M., Oliva, A., 2007, A new Parallel unstructured CFD code for the simulation of turbulent industrial problems on low cost PC cluster, Proceedings of the Parallel CFD 2007 Conference, pp.1-8.

[27] Li, J., Renardy, Y.Y., Renardy, M., 2000. Numerical simulation of breakup of a viscous drop in simple shear flow through a volume-offluid method, Physics of fluids 12, 269-282. 
[28] López, J., Gómez, P., Hernández, J., Faura, F., 2013. A two-grid adaptive volume of fluid approach for dendritic solidification, Computers \& Fluids. 86, 326-342.

[29] Olsson, E., Kreiss, G., 2005. A conservative level set method for two phase flow, J. Comput. Phys. 210, 225-246.

[30] Osher, S., Sethian, J.A., 1988. Fronts propagating with curvaturedependent speed: Algorithms based on Hamilton-Jacobi formulations, J. Comput. Phys. 79, 175-210.

[31] Peskin, C.S., 1977. Numerical analysis of blood flow in the heart, J. Comput. Phys. 25, 220-252.

[32] Raesi, M., Mostaghimi, J., Bussmann, M., 2010. A volume-of-fluid interfacial flow solver with advected normals, Computers \& Fluids 39, 1401-1410.

[33] Renardy, Y., Renardy, M., 2002. PROST: A Parabolic Reconstruction of Surface Tension for the Volume-of-Fluid Method, Journal of Computational Physics 183, 400421.

[34] Rodrigue, D., 2001. Generalized correlation for bubble motion, AIChE Journal. 47, 39-44.

[35] Ryskin, G., Leal, L.G., 1984. Numerical solution of free-boundary problems in fluid mechanics. Part 2. Buoyancy-driven motion of a gas bubble through a quiescent liquid, J. Fluids Mech. 148, 19-35. 
[36] Taylor, G.J., 1934. The Formation of Emulsions in Definable Fields of Flow, Proc. R. Soc. Lond 146, 501-523.

[37] Yue, P., Feng, J.J., Bertelo, C.A., Hu, H.H., 2007. An arbitrary Lagrangian-Eulerian method for simulating bubble growth in polymer foaming, J. Comput. Phys. 226, 2229-2249.

[38] Parker, B., Youngs, D., 1992. Two and three dimensional Eulerian simulation of fluid flow with material interfaces. Technical report 01/92, UK Atomic Weapons Establishment

[39] Rybczynski, W., 1911. Uber die fortschreitende bewegung einer flssigen kugel in einem zhen medium. Bull. Acad. Sci. Cracovi, Ser. A 40

[40] Rhie, C.M., Chow, W.L., 1983. Numerical Study of the Turbulent Flow past an Airfoil with Trailing Edge Separation, AIAA J. 21, 1525-1532.

[41] Sun, D.L., Tao, J., W.Q., 2010. A coupled volume-of-fluid and levelset (VOSET) method for computing incompressible two-phase flows, International Journal of Heat and Mass Transfer 53, 645-655.

[42] Sussman, M., Smereka, P., Osher, S., 1994. A Level Set Approach for Computing Solutions to Incompressible Two-Phase Flow, J. Comput. Phys. 144, 146-159.

[43] Sussman, M., Puckett, E.G., 2000. A Coupled Level Set and Volumeof-Fluid Method for Computing 3D and Axisymmetric Incompressible Two-Phase Flows, J. Comput. Phys. 162, 301-337. 
[44] Sweby, P.K., 1984. High Resolution Using Flux Limiters for Hyperbolic Conservation Laws, SIAM Journal on Numerical Analysis 21, 995-1011.

[45] Taylor, G.I., 1934. The deformation of emulsions in definable fields of flows, Proc. R. Soc. Lond. A 146, 501-523

[46] Tryggvason, G., Bunner, B., Esmaeeli, A., Juric, D., Al-Rawahi, N., Tauber, W., Han, J., Nas, S., Jan, Y-J., 2001. A Front-Tracking Method for the Computations of Multiphase Flow, J. Comput. Phys. 169, 708759.

[47] Unverdi, S., Tryggvason, G., 1992. A front-tracking method for viscous, incompressible, multifluid flows, J. Comput. Phys. 100, 25-37.

[48] Van Sint Annaland, M., Deen, N.G., Kuipers, J.A.M., 2005. Numerical Simulation of gas bubbles behaviour using a three-dimensional volumeof-fluid method, Chemical Engineering Science 60, 2999-3011.

[49] Wang, Z., Yang, J., Koo, B., Stern, F., 2009. A coupled level set and volume-of-fluid method for sharp interface simulation of plunging breaking waves, International Journal of Multiphase Flow 35, 227-246.

[50] Yang, X., James, A., Lowengrub, J., Zheng, X., Cristini, V., 2006. An adaptive coupled level-set/volume-of-fluid interface capturing method for unstructured triangular grids, Journal of Computational Physics 217, 364-394. 
[51] Zahedi, S., Kronbichler, M., Kreiss, G., 2011. Spurious currents in finite element based level set methods, Int. J. Numer. Meth. Fluids, 25-37.

[52] Rallison, J.M., 1981. A numerical study of the deformation and burst of a viscous drop in general shear flows, J. Fluid Mech. 109, 465482.

[53] Kennedy, M.R., Pozrikidis, C., Skalak, R., 1994. Motion and deformation of liquid drops, and the rheology of dilute emulsions in simple shear flow, Computers \& Fluids. 23, 251278.

[54] Komrakova, A.E., Shardt, O., Eskin, D., Derksen, J.J., 2014. Lattice Boltzmann simulations of drop deformation and breakup in shear flow, Int. J. Multiphase Flow 59, 24-43. 